\title{
Diffusion de la lumière par les rugosités d'interface et les hétérogénéités de volume. \\ Application à la caractérisation de microstructure dans les composants interférentiels.
}

Claude Amra

Institut FRESNEL UMR-CNRS 6133

Sciences et Technologies de l'Optique, l'Électromagnétisme et l'Image E.N.S.P.M., F-13397 MARSEILLE, FRANCE

claude.amra@fresnel.fr

\section{INTRODUCTION}

On sait bien, de façon classique en milieu homogène, décrire la trajectoire rectiligne que subit le barycentre de tout faisceau lumineux (Fig. 1-a). Si le milieu supporte un gradient d'indice de réfraction, l'équation eykonale peut être utilisée pour décrire la trajectoire gauche que subit alors le faisceau dans l'espace (Fig. 1-b). Enfin, lorsque les variations d'indice sont aléatoires, une partie de la lumière est perdue, diffusée ou diffractée en dehors de la trajectoire moyenne : c'est la diffusion volumique (Fig. 1-c) ou particulaire, souvent introduite comme à l'origine du bleu du ciel. Cette diffusion permet aussi de visualiser la trace d'un faisceau laser en espace libre dans l'air ou dans un liquide, ou dans un substrat diffusant de type $\mathrm{MgF}_{2}$ ou Zero Dur...

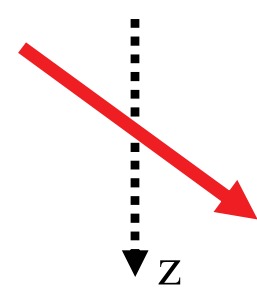

FIG. 1-a. Propagation en milieu homogène.

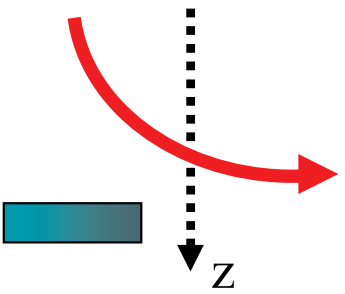

FIG. 1-b. Propagation avec gradient d'indice.

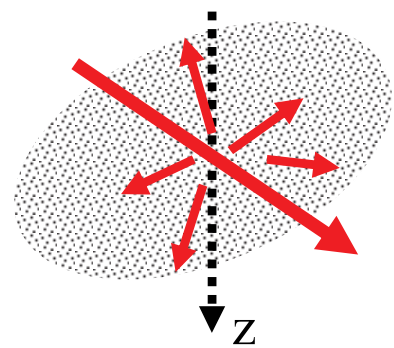

FIG. 1-c.

Indice aléatoire: diffusion volumique.

Cristaux massifs et en couches minces pour l'optique. Élaboration et caractérisation.

C EDP Sciences, 2005

http://dx.doi.org/10.1051/bib-sfo:20028032 
De façon tout aussi classique, en présence d'une interface plane à la séparation de 2 milieux homogènes, les directions de réflexion et transmission de la lumière sont données par les lois de Descartes (Fig. 1-d) :

$$
\mathrm{n} \text { sini }=\text { constante }
$$

avec i l'incidence d'éclairement dans le milieu d'indice $\mathrm{n}$ supposé réel. Les énergies réfléchie et transmise s'obtiennent ensuite par les relations de Fresnel. Le cas des réseaux de diffraction intervient (Fig. 1-e) lorsque la surface de séparation est décrite par une fonction périodique; on assiste alors à une discrétisation de l'énergie dans les demi-espaces avant et arrière, sous forme d'ordres de diffraction dans des directions données en incidence normale $\left(\mathrm{i}=0^{\circ}\right)$ par la loi des réseaux :

$$
\mathrm{n} \sin \theta_{\mathrm{m}}=\mathrm{m} \lambda / \mathrm{C}
$$

avec $\theta$ l'angle normal, $\lambda$ la longueur d'onde, $C$ le pas du réseau et $m$ un nombre entier. On remarquera d'emblée ici, pour annoncer les effets de bande passante, qu'un réseau sub-lambda $(C<\lambda)$ ne diffracte pas et renvoie donc la lumière dans les directions spéculaires (ordres «zéro ») données par les lois de Descartes.

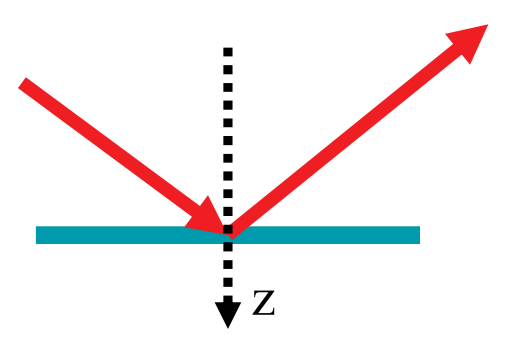

FIG. 1-d.

Réflexion sur une interface plane.

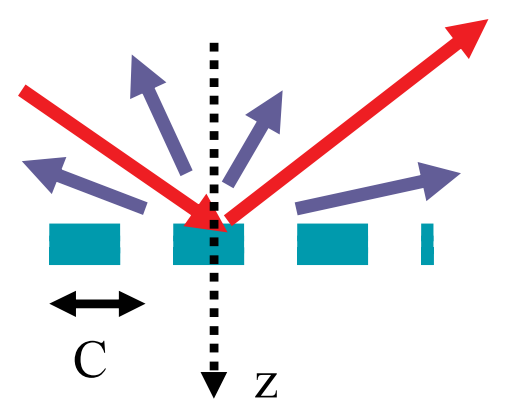

FIG. 1-e. Diffraction par une surface périodique.

Enfin, si l'interface est décrite par une fonction aléatoire, l'énergie incidente est ré-émise de façon continue dans toutes les directions de l'espace (Fig. 1-f), sous forme d'une indicatrice de diffusion : c'est la diffusion de surface, qui permet de visualiser, par exemple, l'impact d'un faisceau laser sur un dioptre. Cette diffusion peut ainsi être considérée comme la diffraction par une infinité de réseaux. De façon générale et quant au vocabulaire utilisé, le terme diffraction est largement utilisé pour le cas d'objets isolés, ou de distribution régulière d'objets déterministes, alors que la diffusion est souvent liée au cas de surfaces ou volumes aléatoires.

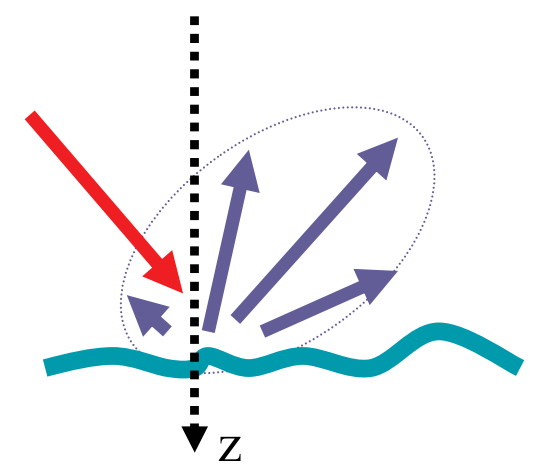

FIG. 1-f.

Diffusion par une surface aléatoire. 
Pour conclure, on insistera sur le paramètre clé qui est la fréquence spatiale $(v=n \sin \theta / \lambda)$ associée aux directions réfléchie et transmise, diffractées ou diffusées. En effet :

- $\quad \mathrm{v}=\mathrm{v}_{0}=$ constante

est une relation qui traduit les lois de Descartes, avec la fréquence incidente $v_{0}=n \operatorname{sini} / \lambda$;

- $\quad v=v_{m}=m v_{g}$

décrit la loi des réseaux en incidence normale, avec $v_{\mathrm{g}}=1 / \mathrm{C}$ la fréquence du réseau ;

- $\quad v=$ réel arbitraire (variable de Fourier)

est une propriété caractéristique d'une surface aléatoire puisqu'elle indique qu'il y a diffusion dans chaque direction $\theta$ de l'espace.

Enfin, on se limitera aux basses fréquences $\left(v<\mathrm{n} \lambda^{-1}\right)$ caractéristiques des ondes planes transportant de l'énergie en champ lointain. Au delà de $n \lambda^{-1}$, les hautes fréquences associées aux ondes évanescentes décrivent la structure fine du champ proche. Ces ondes peuvent éventuellement être découplées si l'on modifie le milieu superstrat.

Grâce à leur simplicité, les formules scalaires ont rendu de nombreux services aux opticiens. Nous montrons ci-dessous comment retrouver, à l'aide de considérations de base mettant en jeu quelques notions statistiques et de chemin optique, la formule très connue dite du TIS (Total Integrated Scattering) ou diffusion globale (réflexion ou transmission diffuse).

\subsection{Paramètres statistiques}

Considérons une surface décrite par la fonction $z=h(\mathbf{r})$, avec $\mathbf{r}=(\mathrm{x}, \mathrm{y})$. Traçons maintenant des droites perpendiculaires à l'axe z (Fig. 2) et comptons, à chaque altitude z, le nombre d'occurrences où ces droites interceptent la surface.

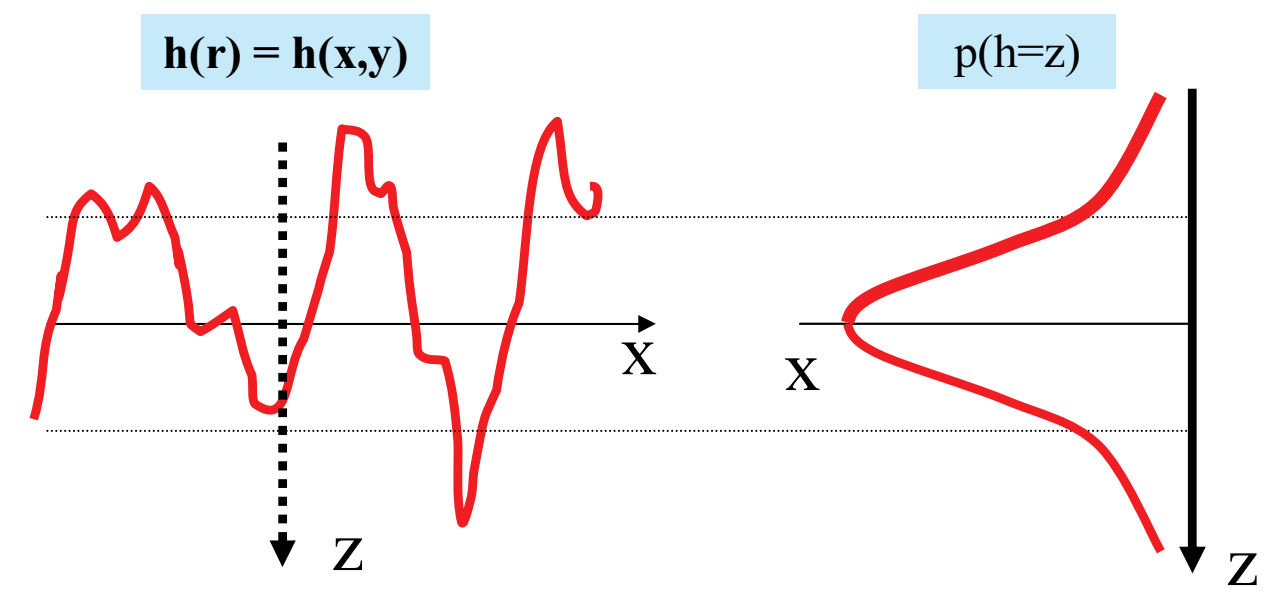

FIG. 2. Projection $\mathrm{p}(\mathrm{h}=\mathrm{z}) \mathrm{d}$ 'une surface aléatoire $\mathrm{h}(\mathrm{x}, \mathrm{y})$. 
On obtient ainsi une projection ou fréquence $\mathrm{p}(\mathrm{h}=\mathrm{z})$ qui est un estimateur de la densité de probabilité de la surface. Pour une surface gaussienne, on aurait donc :

$$
\mathrm{p}(\mathrm{z})=\alpha \exp \left[-(\mathrm{z} / \mathrm{L})^{2}\right], \text { avec } \alpha=1 /(\mathrm{L} \sqrt{ } \pi)
$$

pour normaliser la loi de probabilité.

Cette loi (ㅁ) étant établie, on peut définir les différents moments ou paramètres statistiques de la surface supposée ici ergodique :

- La moyenne m est décrite par :

$$
\mathrm{m}=<\mathrm{h}>=\int_{\mathrm{z}} \mathrm{z} \mathrm{p}(\mathrm{h}=\mathrm{z}) \mathrm{dz}
$$

où le symbole $\diamond$ définit une moyenne d'ensemble. On considérera que cette moyenne est nulle, ce qui revient à centrer la surface ou fixer l'origine des z.

- L'écart-type $\delta$ décrit la distance moyenne de la surface par rapport au plan moyen $(z=0)$ :

$$
\delta^{2}=<(\mathrm{h}-\mathrm{m})^{2}>=<\mathrm{h}^{2}>=\int_{\mathrm{z}} \mathrm{z}^{2} \mathrm{p}(\mathrm{h}=\mathrm{z}) \mathrm{dz}
$$

Dans le cas d'une surface gaussienne, on obtient : $\delta=\mathrm{L} / \sqrt{ } 2$. Cette grandeur sera souvent appelée rugosité, analogue à une hauteur quadratique moyenne.

- La fonction caractéristique est une moyenne d'exponentielle liée au profil :

$$
\chi(\beta)=<\exp (j \beta z)>=\int_{z} e^{j \beta z} p(h=z) d z
$$

Cette fonction n'est autre, à un coefficient près, que la transformée de Fourier $p(\beta)$ de la densité de probabilité $\mathrm{p}(\mathrm{z})$ :

$$
\begin{array}{ll} 
& \chi(\beta)=2 \pi p(\beta) \\
\text { avec : } & p(\beta)=(1 / 2 \pi) \int_{z} \mathrm{p}(\mathrm{z}) \mathrm{e}^{-\mathrm{j} \beta z} \mathrm{dz} \\
\text { et : } & \mathrm{p}(\mathrm{z})=\int_{\mathrm{z}} p(\beta) \mathrm{e}^{\mathrm{j} \beta z} \mathrm{dz}
\end{array}
$$

Pour une statistique gaussienne, on obtient :

$$
\chi(\beta)=\exp \left[-(\beta \mathrm{L} / 2)^{2}\right]
$$

\subsection{Champ réfléchi}

Considérons une onde plane d'amplitude

$$
\mathrm{E}_{0}^{+}=\mathrm{A}_{0}^{+} \exp \left[\mathrm{j}\left(\sigma \mathrm{x}+\alpha_{0} \mathrm{z}\right)\right]
$$

interagissant avec la surface rugueuse sous l'incidence (i) dans le milieu incident d'indice $\left(\mathrm{n}_{0}\right)$ (Fig. 3-a). 


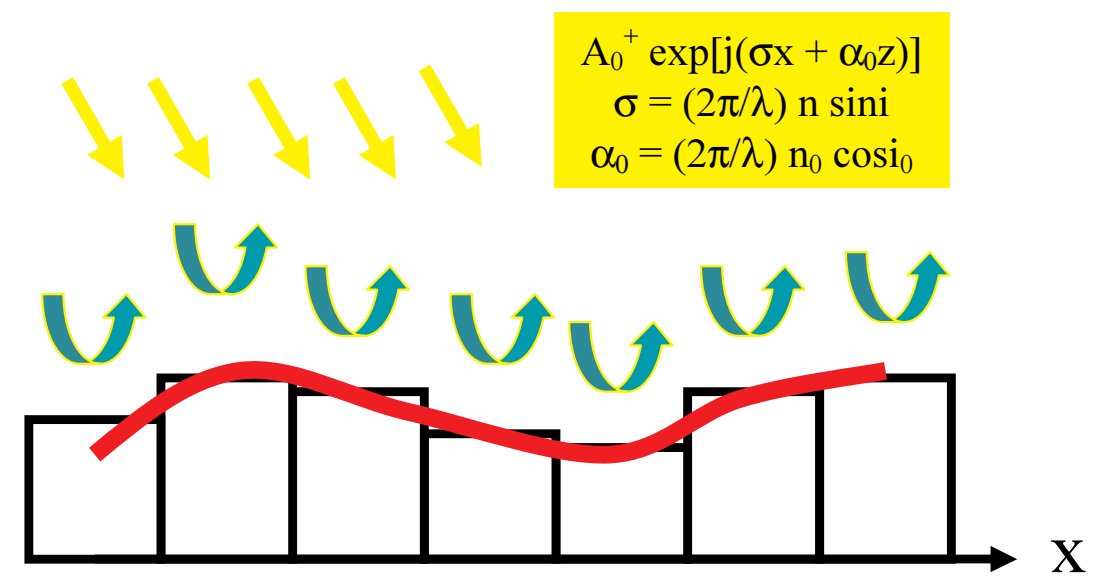

FIG. 3-a. Approximation de la fonction par une surface en escalier.

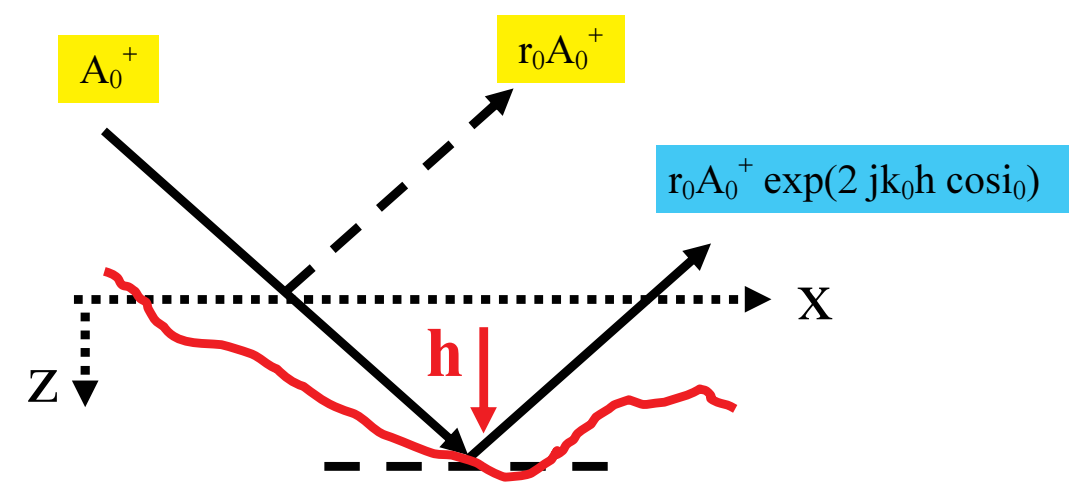

FIG. 3-b. Déphasage entre les ondes réfléchies par le dioptre rugueux et le dioptre plan.

Les paramètres du vecteur d'onde sont donnés par :

$$
\sigma / 2 \pi=v=n \operatorname{sini} / \lambda \text { et } \alpha_{0} / 2 \pi=n_{0} \operatorname{cosi} i_{0} / \lambda
$$

Pour obtenir un résultat immédiat, on peut utiliser l'approximation brutale d'une surface en escalier. Ceci nous amène à considérer l'interaction « lumière/surface rugueuse » comme une simple réflexion locale de rayons à chaque marche de l'escalier, décrite par les lois de Fresnel sur les plans tangents à ces marches. Cette hypothèse est évidemment forte et ne peut être justifiée que pour des surfaces à faibles pentes ou grande longueur de corrélation. Ce point sera précisé en section 4. Pour la suite, on considérera que la surface est à pentes faibles, de sorte que tous les plans tangents sont horizontaux (perpendiculaires à z). la forme :

Comme illustré en figure 3-b , le faisceau réfléchi localement s'écrit dans ces conditions sous

$$
E_{r}=r_{0} E_{0}^{+} \exp \left[2 j k_{0} h \cos i_{0}\right]
$$


où $\mathrm{r}_{0}$ désigne le coefficient de réflexion en amplitude (complexe) de la surface plane, et $\mathrm{k}_{0} / 2 \pi=\mathrm{n}_{0} / \lambda$ la fréquence maximale pour l'espace libre dans le milieu incident. On notera que le terme en exponentielle traduit le déphasage en champ lointain de l'onde réfléchie sur la marche locale, par rapport à l'onde qui serait réfléchie par un dioptre idéalement plan.

Il est alors aisé d'obtenir l'amplitude moyenne de la réflexion :

$$
<\mathrm{E}_{\mathrm{r}}>=\mathrm{r}_{0} \mathrm{E}_{0}^{+} \chi\left(2 \mathrm{k}_{0} \cos \mathrm{i}_{0}\right)
$$

ainsi que l'intensité moyenne $\mathrm{R}$ réfléchie par le dioptre rugueux dans la direction de réflexion spéculaire :

$$
\mathrm{R}=\mathrm{R}_{0}\left|\chi\left(2 \mathrm{k}_{0} \cos \mathrm{i}_{0}\right)\right|^{2}
$$

avec $\mathrm{R}_{0}$ la réflexion par un dioptre plan. La quantité totale de lumière diffusée, ou réflexion diffuse, s'écrit comme l'écart par rapport à la réflexion spéculaire idéale :

$$
\mathrm{S}=\mathrm{R}_{0}-\mathrm{R}=\mathrm{R}_{0}\left[1-\left|\chi\left(2 \mathrm{k}_{0} \cos \mathrm{i}_{0}\right)\right|^{2}\right]=<\left|\mathrm{E}_{\mathrm{r}}-<\mathrm{E}_{\mathrm{r}}>\right|^{2}>
$$

Dans le cas d'un faisceau gaussien, on retrouve ainsi les formules très connues suivantes :

$$
\mathrm{R}=\mathrm{R}_{0} \exp \left[-\left(2 \mathrm{k}_{0} \delta \operatorname{cosi}_{0}\right)^{2}\right] \text { et } \mathrm{S}=\mathrm{R}-\mathrm{R}_{0}
$$

Pour une surface faiblement rugueuse, définie comme :

$$
2 \mathrm{k}_{0} \delta \operatorname{cosi}_{0}<<1 \Leftrightarrow \delta \operatorname{cosi}_{0}<<\lambda
$$

on obtient, après développement limité au premier ordre :

$$
\mathrm{S} / \mathrm{R}=\left(4 \pi \mathrm{n}_{0} \delta \cos \mathrm{i}_{0} / \lambda\right)^{2}
$$

Dans la littérature anglo-saxonne, ce dernier rapport est souvent désigné par TIS (Total Integrated Scattering).

\subsection{Cas de la transmission}

La figure 3-c est donnée pour le cas de la transmission. Un raisonnement analogue conduit à obtenir le champ transmis et sa moyenne comme :

$$
\mathrm{E}_{\mathrm{t}}=\mathrm{t} \mathrm{E}_{0}^{+} \exp \left(\mathrm{j} \alpha_{0} \mathrm{~h}\right) \exp \left(-\mathrm{j} \alpha_{1} \mathrm{~h}\right) \text { et }<\mathrm{E}_{\mathrm{t}}>=\mathrm{tE}_{0}^{+} \chi\left(\alpha_{0}-\alpha_{1}\right)
$$

avec $\alpha_{i} / 2 \pi=n_{i} \operatorname{cosi} i_{i} / \lambda$, et $n_{0}$ et $n_{1}$ sont les indices des superstrat et substrat. L'intensité moyenne vaut alors :

$$
\mathrm{T}=\mathrm{T}_{0} \exp \left[-\left(\alpha_{0}-\alpha_{1}\right)^{2} \delta^{2}\right]
$$

avec $\mathrm{T}_{0}$ la transmission du dioptre plan. La transmission diffuse s'écrit : $\mathrm{S}_{\mathrm{T}}=\mathrm{T}_{0}-\mathrm{T}$ et vaut, pour une surface faiblement rugueuse :

$$
\mathrm{S}_{\mathrm{T}}=\mathrm{T}_{0}\left(\mathrm{n}_{1} \operatorname{cosi} \mathrm{i}_{1}-\mathrm{n}_{0} \cos \mathrm{i}_{0}\right)^{2}(2 \pi \delta / \lambda)^{2}
$$


Cette expression ne doit pas laisser croire que la transmission diffuse est proportionnelle au facteur de transmission idéal $\mathrm{T}_{0}$, par analogie à la réflexion diffuse qui est proportionnelle à $\mathrm{R}_{0}$. En fait le rapport des énergies diffusées en réflexion et transmission est donné par le rapport des indices de substrat et superstrat : $\mathrm{S}_{\mathrm{T}} / \mathrm{S}_{\mathrm{R}}=\mathrm{n}_{1} / \mathrm{n}_{0}(25)$, qui est de l'ordre de grandeur de l'unité.

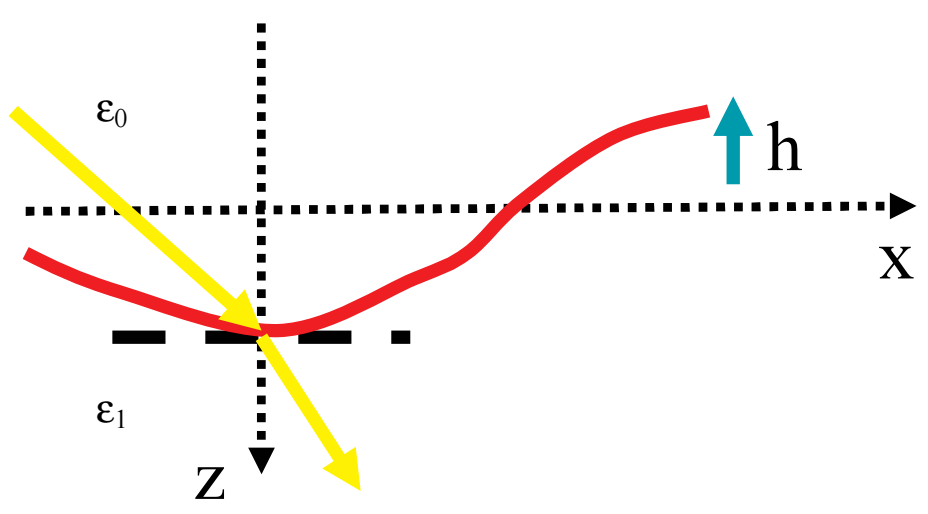

FIG. 3-c. Cas de la transmission.

\subsection{Conclusion}

Résumons ci-dessous les résultats-clés qui peuvent être utilisés directement pour obtenir l'ordre de grandeur d'une diffusion de surface :

- Les facteurs de réflexion et de transmission spéculaires d'une interface sont affectés par la présence de rugosité à cette interface ; en lumière monochromatique et pour une surface à faibles pentes, ces modifications sont décrites par les formules suivantes :

$$
\left.\mathrm{R}=\mathrm{R}_{0} \exp \left[-\left(2 \alpha_{0} \delta\right)^{2}\right] \quad \mathrm{T}=\mathrm{T}_{0} \exp \left[-\left(\alpha_{0}-\alpha_{1}\right) \delta\right)^{2}\right]
$$

où $\mathrm{R}_{0}, \mathrm{~T}_{0}$ et $\mathrm{R}, \mathrm{T}$ désignent les facteurs de réflexion et de transmission de la surface plane et de la surface rugueuse, respectivement. $\delta$ désigne la rugosité et le paramètre $\alpha$ est donné par $\alpha_{i} / 2 \pi=n_{i} \operatorname{cosi} i_{i} / \lambda$, où $n_{i}$ et $i_{i}$ sont les indices et angles d'incidence des (ou dans) les milieux superstrat $\left(\mathrm{n}_{0}, \mathrm{i}_{0}\right)$ et substrat $\left(\mathrm{n}_{1}, \mathrm{i}_{1}\right)$.

- Parallèlement, les réflexion $\left(\mathrm{S}_{\mathrm{R}}\right)$ et transmission $\left(\mathrm{S}_{\mathrm{T}}\right)$ diffuses que l'on peut mesurer en espace libre s'écrivent comme :

$$
\mathrm{S}_{\mathrm{R}}=\Delta \mathrm{R}=\mathrm{R}_{0}-\mathrm{R} \quad \mathrm{S}_{\mathrm{T}}=\Delta \mathrm{T}=\mathrm{T}_{0}-\mathrm{T}
$$

avec $\mathrm{S}_{\mathrm{T}} / \mathrm{S}_{\mathrm{R}}=\mathrm{n}_{1} / \mathrm{n}_{0}$. Si la surface est faiblement rugueuse, ces expressions deviennent :

$$
\mathrm{S}_{\mathrm{R}}=\mathrm{R}_{0}\left(2 \alpha_{0}\right)^{2} \delta^{2} \quad \text { et } \quad \mathrm{S}_{\mathrm{T}}=\mathrm{T}_{0}\left(\alpha_{0}-\alpha_{1}\right)^{2} \delta^{2}
$$

soit encore : $\quad \mathrm{S}_{\mathrm{R}}=\mathrm{R}_{0}\left(4 \pi \mathrm{n}_{0} \delta \cos \mathrm{i}_{0} / \lambda\right)^{2}$

$$
\mathrm{S}_{\mathrm{T}}=\mathrm{T}_{0}\left(\mathrm{n}_{1} \operatorname{cosi} \mathrm{i}_{1}-\mathrm{n}_{0} \cos \mathrm{i}_{0}\right)^{2}(2 \pi \delta / \lambda)^{2}
$$


- Enfin, pour chiffrer les ordres de grandeur, on peut remarquer que :

$$
\begin{aligned}
\mathrm{R} / \mathrm{R}_{0} & =\exp \left[-16 \pi^{2}\left(\mathrm{n}_{0} \operatorname{cosi}_{0} \delta / \lambda\right)^{2}\right] \\
& =10^{-70} \operatorname{pour} \delta=\lambda \\
& =0,2 \text { pour } \delta / \lambda=0,1 \\
& =0,98 \text { pour } \delta / \lambda=0,01
\end{aligned}
$$

Ainsi la réflexion spéculaire classique est absente lorsque la rugosité est proche ou au delà de la longueur d'onde, ce qui est le cas d'une majorité de surfaces (murs, peintures, baffleurs, dépolis, textiles, tissus biologiques...). À l'inverse, les surfaces optiques (polies) diffusent une quantité de lumière toujours inférieure au pour cent. De façon plus précise, on sait aujourd'hui fabriquer des superpolis avec des rugosités égales ou inférieures à l'Angström (dans la bande passante optique$c f$. $\underline{\text { section } 7)}$; la diffusion globale de ces superpolis est inférieure au millionième $\left(10^{-6}\right)$ du flux incident.

Soulignons le fait que ces lumières diffuses (en réflexion et transmission) sont proportionnelles au facteur de réflexion de l'échantillon plan. Ainsi, à rugosité égale, une surface d'Aluminium diffuse environ 20 fois plus qu'une surface de verre dans le visible. Il est également utile de savoir que ces formules scalaires s'appliquent aux systèmes multicouches, à condition que toutes les interfaces du multicouche soient parfaitement corrélées. On en déduit que la diffusion spectrale (variations avec la longueur d'onde) d'un multicouche corrélé varie comme le facteur de réflexion de l'échantillon. Cette propriété est souvent utilisée pour discriminer des effets de surface ou volume, ou pour étudier l'état d'intercorrélation... On en déduit également que la diffusion peut être exaltée ou réduite par le dépôt d'un système multicouche, selon la corrélation entre interfaces, ou selon la formule du composant. Enfin, pour les systèmes multicouches, il faut considérer la répartition intra-cavité du champ excitateur stationnaire, qui peut créer des résonances fortes comme dans le cas des Fabry-Pérot.

Pour conclure cette partie, et malgré l'extrême utilité de ces formules scalaires, il nous faut souligner, en deçà du domaine de validité des hypothèses, une autre faiblesse liée à cette modélisation scalaire, qui ne met pas en évidence les effets de bande passante. En particulier on ne peut pas parler de rugosité ou de microstructure si l'on ne précise pas l'échelle de fréquences spatiales mises en jeu, qui peut aller du microscopique au macroscopique. Pour résoudre ce point, nous présentons maintenant une théorie électromagnétique prenant en compte la variation angulaire de diffusion.

\section{APPROCHE DÉTERMINISTE : MODÈLE ÉLECTROMAGNÉTIQUE}

Bien que limités au premier ordre, les modèles basés sur l'approximation de Born sont souvent incontournables, pour les raisons suivantes:

- ils donnent un accès quasi-immédiat à la diffusion angulaire, dans le plan d'incidence et en dehors de ce plan ;

- ce sont des modèles bi ou tri-dimensionnels, c'est-à-dire que les objets diffusants sont décrits par des fonctions de une ou deux variables ;

- les effets de polarisation ou dépolarisation sont accessibles par ces modèles dans tout l'espace ;

- leur application et généralisation aux multicouches est immédiate ;

- ils s'appliquent également aux guides d'onde multicouche plans ; 
- ils s'appliquent au contrôle de l'émission spontanée dans les microcavités planaires, pour une interaction faible entre la source et les champs ;

- enfin, de par leur simplicité analytique, ces théories sont souvent utilisées au préalable pour synthétiser les composants fortement diffractants dans le but d'exalter, d'inhiber ou d'orienter les flux diffusés. Ces solutions initiales sont ensuite testées ou éprouvées grâce à des codes de calcul exact (méthodes différentielles, intégrales ou modales...) qui prennent alors le relai, avec une complexité et des temps de calcul bien plus grands.

\subsection{Paramètres déterministes}

La surface est maintenant décrite par l'équation $\mathrm{z}=\mathrm{h}(\mathbf{r})$ avec $\mathbf{r}=(\mathrm{x}, \mathrm{y})$, comme illustré en figure 4. Sa normale orientée dans le sens des z $>0$ s'écrit :

$$
\mathbf{n}=(\mathbf{z}-\operatorname{grad} \mathrm{h})\left[1+(\operatorname{grad} \mathrm{h})^{2}\right]^{-1 / 2}
$$

On considère à nouveau que le profil est centré, ce qui revient à écrire que la moyenne est nulle :

$$
\hbar=(1 / S) \int_{\mathbf{r}} h(\mathbf{r}) \mathrm{d} \mathbf{r}=0
$$

où $\mathrm{S}$ désigne l'aire de la zone éclairée. L'écart-type ou carré de la rugosité est donné par l'écart quadratique moyen :

$$
\delta^{2}=(1 / \mathrm{S}) \int_{\mathbf{r}} \mathrm{h}^{2}(\mathbf{r}) \mathrm{d} \mathbf{r}
$$

Enfin, la fonction d'autocorrélation ou d'autocovariance, qui est une mesure de l'ordre ou du désordre sur la surface, s'écrit :

$$
\Gamma(\tau)=(1 / \mathrm{S}) \int_{\mathbf{r}} \mathrm{h}(\mathbf{r}) \mathrm{h}(\mathbf{r}-\tau) \mathrm{d} \mathbf{r} \quad \text { avec } \tau=\left(\tau_{\mathrm{x}}, \tau_{\mathrm{y}}\right) \text { et } \Gamma(\mathbf{0})=\delta^{2}
$$

Rappelons que, en dehors des effets de fonction d'appareil, tout extrema de cette fonction traduit la présence d'une pseudo-périodicité sur la surface.

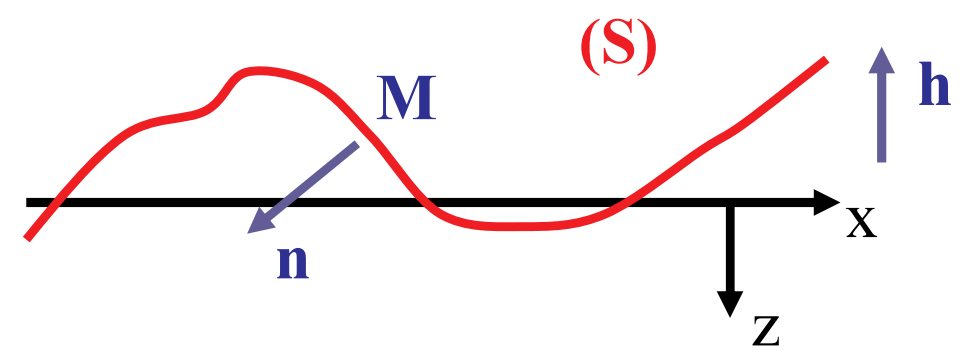

FIG. 4. Surface rugueuse (S) d'équation $\mathrm{z}=\mathrm{h}(\mathrm{r})$.

Nous aurons également besoin du spectre de rugosité, ou densité spectrale de puissance, défini comme :

$$
\gamma(\sigma)=\left(4 \pi^{2} / \mathrm{S}\right)|h(\sigma)|^{2}
$$

où $h(\sigma)$ désigne la transformée de Fourier du profil h(r) :

$$
\begin{array}{ll} 
& h(\boldsymbol{\sigma})=\left(1 / 4 \pi^{2}\right) \int_{\mathbf{r}} \mathrm{h}(\mathbf{r}) \mathrm{e}^{-\mathrm{j} \boldsymbol{\sigma} . \mathbf{r}} \mathrm{d} \mathbf{r} \\
\text { avec : } & \mathrm{h}(\mathbf{r})=\int_{\boldsymbol{\sigma}} h(\boldsymbol{\sigma}) \mathrm{e}^{\mathrm{j} \boldsymbol{\sigma} . \mathbf{r}} \mathrm{d} \boldsymbol{\sigma}
\end{array}
$$


Remarquons ici que la TF est bi-dimensionnelle, ce qui explique l'apparition de termes en $4 \pi^{2}$, ainsi qu'une pulsation spatiale de type vecteur :

$$
\boldsymbol{\sigma}=\left(\sigma_{\mathrm{x}}, \sigma_{\mathrm{y}}\right)
$$

qui nous permettra de définir les angles normaux et polaires de diffusion. Précisons également que fréquence et pulsation sont liés par :

$$
\sigma=2 \pi v
$$

avec $\boldsymbol{v}$ la fréquence spatiale. Enfin, pour utilisation des TF, rappelons que tout pic présent dans le spectre de l'échantillon traduit la présence, dans le profil de surface, d'une harmonique ou fonction périodique.

La rugosité est souvent obtenue par intégration du spectre de rugosité, qui sera la grandeur directement accessible par l'expérience :

$$
\delta^{2}=\Gamma(\mathbf{0})=\int_{\boldsymbol{\sigma}} \gamma(\boldsymbol{\sigma}) \mathrm{d} \boldsymbol{\sigma}=(1 / \mathrm{S}) \int_{\mathbf{r}} \mathrm{h}^{2}(\mathbf{r}) \mathrm{d} \mathbf{r}
$$

En faisant intervenir le Jacobien de l'intégrale, on obtient :

$$
\delta^{2}=2 \pi \int_{\sigma} \sigma \gamma_{\mathrm{m}}(\sigma) \mathrm{d} \sigma
$$

avec :

$$
\gamma_{\mathrm{m}}(\sigma)=(1 / 2 \pi) \int_{\phi} \gamma(\sigma, \phi) \mathrm{d} \phi
$$

Dans cette dernière expression, $\gamma_{\mathrm{m}}$ désigne un spectre moyen, ou moyenne polaire du spectre, l'angle polaire $\phi$ étant défini par :

$$
\boldsymbol{\sigma}=\sigma(\cos \phi, \sin \phi) \quad \operatorname{avec} \sigma=|\sigma|
$$

Il est également intéressant de souligner les dimensions de ces grandeurs; en effet, certains appareils sont adaptés à la mesure d'objets uni- ou bi-dimensionnels (1 ou 2 variables), d'où l'utilité de préciser que :

- dans le cas $1 \mathrm{D}, \mathrm{h}(\mathbf{r})$ est en $[\mathrm{m}], \Gamma(\tau)$ et $\delta^{2}$ sont en $[\mathrm{m}]^{2}, h(\sigma)$ en $[\mathrm{m}]^{2}$ et $\gamma(\sigma)$ en $[\mathrm{m}]^{3}$;

- dans le cas 2D, h(r) est en $[\mathrm{m}], \Gamma(\tau)$ et $\delta^{2}$ sont en $[\mathrm{m}]^{2}, h(\sigma)$ en $[\mathrm{m}]^{3}$ et $\gamma(\sigma)$ en $[\mathrm{m}]^{4}$.

\section{2. Équations locales sur la surface rugueuse}

En régime harmonique ou monochromatique, les équations de Maxwell s'écrivent, pour un dioptre idéalement plan et non chargé (Fig. 5-a), comme :

$$
\begin{aligned}
& \operatorname{rot} \mathbf{E}_{\mathbf{i}}^{0}=j \omega \mu \mathbf{H}_{i}^{0} \\
& \operatorname{rot} \mathbf{H}_{i}^{0}=-j \omega \varepsilon_{i} \mathbf{E}_{\mathbf{i}}^{0}
\end{aligned}
$$

où $\mathbf{E}_{\mathbf{i}}{ }^{0}, \mathbf{H}_{\mathbf{i}}{ }^{0}$ désignent les champs spéculaires (complexes) classiques dans le milieu d'indice $\left(\mathrm{n}_{\mathrm{i}}\right)$. Ces équations sont valables pour tout milieu homogène, isotrope, linéaire et non magnétique. 
Considérons maintenant le cas de la surface rugueuse, comme illustré en figure 5-b. On définit localement le champ diffusé $\mathbf{E}^{\mathbf{d}}$ comme une perturbation donnée par :

$$
\mathbf{E}^{\mathbf{d}}=\mathbf{E}-\mathbf{E}^{\mathbf{0}}
$$

où $\mathbf{E}$ désigne le champ total ré-émis par la surface rugueuse.

Les champs idéal $\left(\mathbf{E}^{\mathbf{0}}\right)$ et total $(\mathbf{E})$ vérifiant les équations de Maxwell, il en est de même du champ diffusé :

$$
\begin{aligned}
& \operatorname{rot} \mathbf{E}_{i}^{d}=j \omega \mu \mathbf{H}_{i}^{d} \\
& \operatorname{rot} \mathbf{H}_{i}^{d}=-j \omega \varepsilon_{i} \mathbf{E}_{i}^{d}
\end{aligned}
$$

Ces équations sont valables au sens des fonctions, c'est-à-dire en dehors des sillons de la surface. Pour poser complètement le problème, il faut écrire les relations de passage sur la surface :

$$
\mathbf{n} \wedge \boldsymbol{\delta} \mathbf{E}=\mathbf{n} \wedge \boldsymbol{\delta} \mathbf{H}=\mathbf{0} \quad \text { au point } \mathrm{M}(\mathbf{r}, \mathrm{z}=\mathrm{h}(\mathbf{r}))
$$

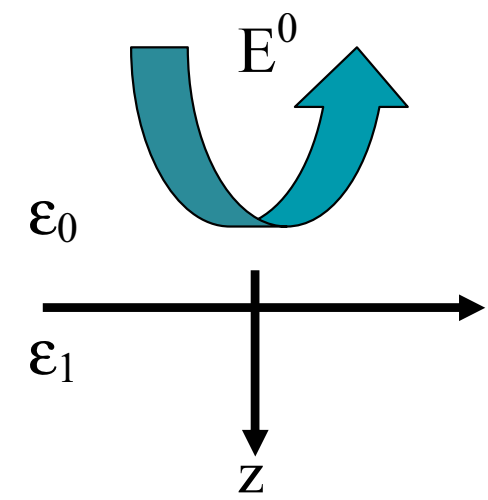

FIG. 5-a. Champ idéal.

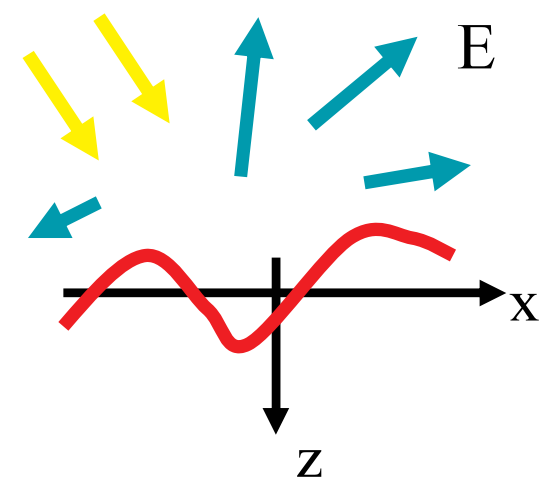

FIG. 5-b. Champ total.

En supposant la surface faiblement rugueuse $(\delta / \lambda<<1)$ et de faibles pentes $(|\operatorname{grad} h|<<1)$, il est aisé d'effectuer un développement limité de ces relations, en ne conservant que les termes du premier ordre. Le résultat est alors donné par :

$$
\mathbf{z}^{\wedge} \boldsymbol{\delta} \mathbf{E}^{\mathbf{d}}=\mathbf{M} \quad \text { et } \quad \mathbf{z}^{\wedge} \boldsymbol{\delta} \mathbf{H}^{\mathrm{d}}=\mathbf{J}
$$

avec : $\quad \mathbf{M}\left(\mathbf{E}^{0}\right)=\operatorname{grad} \mathrm{h} \wedge \delta \mathbf{E}^{0}-\mathrm{h} \mathbf{z} \wedge \delta\left[\partial_{\mathrm{z}} \mathbf{E}^{0}\right]$ et $\mathbf{J}\left(\mathrm{E}^{0}\right)=-\mathrm{h} \mathbf{z} \wedge \delta\left[\partial_{\mathrm{z}} \mathbf{H}^{0}\right]$

Les équations de Mawwell s'écrivent finalement pour le champ diffusé, au sens des distributions, comme :

$$
\begin{aligned}
& \operatorname{rot} \mathbf{E}_{\mathbf{i}}^{\mathbf{d}}=\mathrm{j} \omega \mu \mathbf{H}_{\mathbf{i}}{ }^{\mathbf{d}}+\mathbf{M} \delta(\mathrm{z}) \\
& \operatorname{rot} \mathbf{H}_{\mathbf{i}}{ }^{\mathbf{d}}=-\mathrm{j} \omega \varepsilon_{\mathrm{i}} \mathbf{E}_{\mathbf{i}}{ }^{\mathbf{d}}+\mathbf{J} \delta(\mathrm{z})
\end{aligned}
$$

avec $\delta$ la distribution de Dirac. Ainsi le problème du champ diffusé est identique à celui du champ rayonné par des courants électrique $(\mathbf{J})$ et magnétique $(\mathbf{M})$ de surface, ayant pour support un dioptre 
idéalement plan. Grâce à l'approximation de Born (développement au premier ordre), les sources ou discontinuités $\mathbf{M}$ et $\mathbf{J}$ sont connues puisque seulement excitées par le champ idéal classique $\mathbf{E}^{\mathbf{0}}$.

\subsection{Intensité diffusée}

Le système d'équations $(\underline{48})$ peut être résolu directement dans le plan de Fourier à l'aide de la notion d'admittance complexe. Le champ diffracté $\mathrm{E}^{\mathrm{d}}$ est alors proportionnel à la transformée de Fourier $h(\boldsymbol{\sigma})$ du profil de la surface rugueuse, ce qui est une caractéristique des théories du premier ordre. On en déduit que l'intensité diffusée I se met sous la forme :

$$
\mathrm{I}(\boldsymbol{\sigma})=\mathrm{C}\left(\boldsymbol{\sigma}_{0}, \boldsymbol{\sigma}\right) \gamma\left(\boldsymbol{\sigma}-\boldsymbol{\sigma}_{0}\right)
$$

où $\sigma_{0}$ et $\sigma$ désignent les fréquences spatiales de l'onde incidente et diffusée, respectivement :

$$
\text { - } \quad \sigma_{0} / 2 \pi=(\mathrm{n} \operatorname{sini} / \lambda)(\cos \psi, \sin \psi)
$$

avec i l'angle normal de l'éclairement dans le milieu d'indice n, et $\psi$ l'angle polaire d'éclairement qui définit le plan d'incidence (en général on choisit l'origine comme $\left.\psi=0^{\circ}\right)$;

- $\quad \sigma / 2 \pi=(\mathrm{n} \sin \theta / \lambda)(\cos \phi, \sin \phi)$

permet de définir les angles normaux et polaires de l'onde diffusée dans la direction $(\theta, \phi)$ en champ lointain ( $\underline{\text { Fig. 6) }}$.

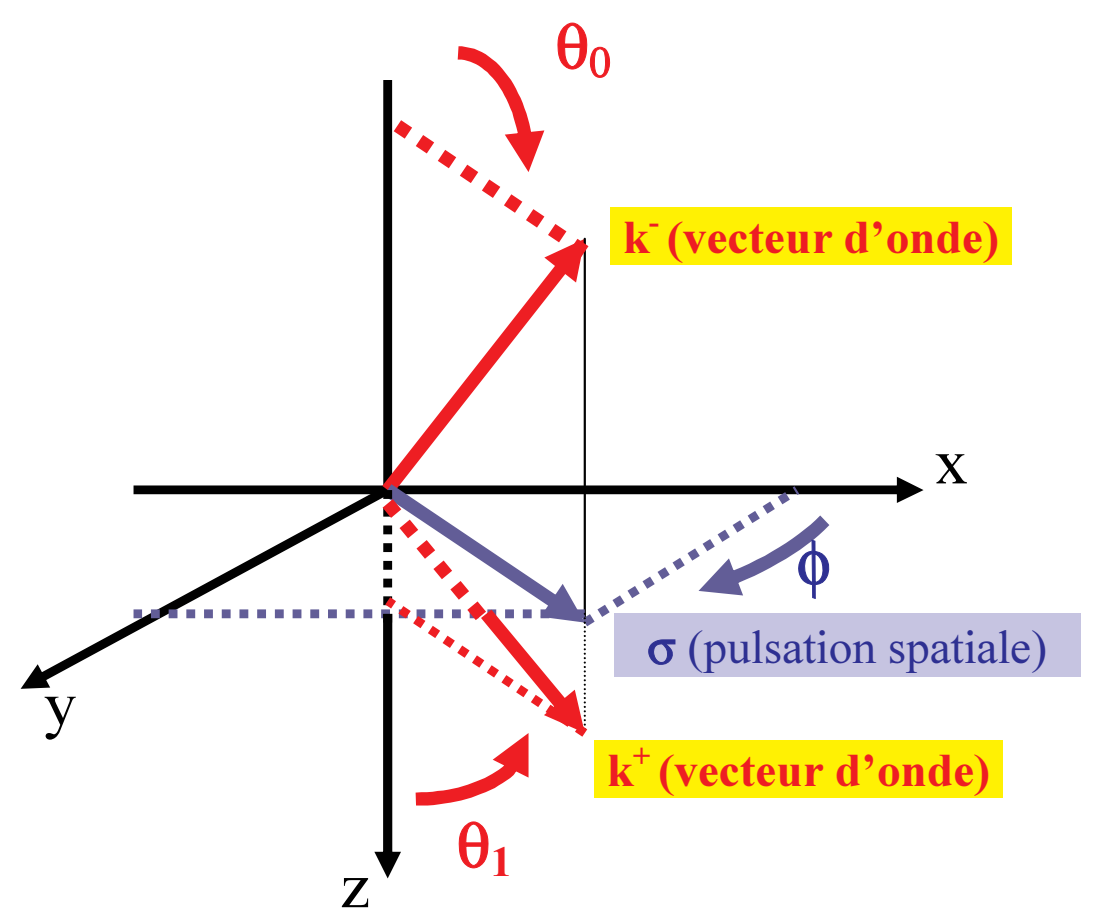

FIG. 6. Définition des angles de diffusion à partir de la fréquence spatiale. 
Quant au coefficient C, qualifié de coefficient idéal ou excitateur parce qu'il dépend du champ $\mathbf{E}^{0}$ qui alimente les courants, il est analogue à une efficacité ou rendement et ne dépend pas de la microgéométrie de la surface, mais uniquement des conditions d'éclairement et d'observation (incidence, longueur d'onde et polarisation), ainsi que des indices des milieux. Ainsi la formule électromagnétique peut être ré-écrite sous la forme :

$$
\mathrm{I}(\theta, \phi)=\mathrm{C}_{\mathrm{i}}(\theta, \phi) \gamma_{\mathrm{i}}(\theta, \phi)
$$

Cette expression va s'avérer très pratique pour la résolution de problèmes inverses, puisque le terme de microstructure (le spectre de rugosité) apparaît comme un simple coefficient multiplicatif facile à isoler. Précisons que l'intensité angulaire I représente le flux diffusé $(\mathrm{d} \Phi / \mathrm{d} \Omega)$ par unité d'angle solide ; il est souvent présenté comme une BRDF (Bidirectional Reflectance Distribution Function) ou une luminance $(\mathrm{L})$, à savoir :

$$
\mathrm{I}=\mathrm{d} \Phi / \mathrm{d} \Omega=\text { BRDF. } \cos \theta=\mathrm{L} \cos \theta
$$

En lumière naturelle et en incidence normale $\left(i=0^{\circ}\right)$, où la dépendance polaire est uniquement imposée par le spectre $\gamma$, nous donnons ci-dessous les éléments pour calculer les facteurs optiques $\mathrm{C}^{-}$et $\mathrm{C}^{+}$relatifs aux diffusions en réflexion et en transmission :

$$
\begin{aligned}
& \mathrm{C}^{-}(\sigma)=\left(\mathrm{k}_{0}{ }^{2} / 2\right)\left[\cos ^{2} \theta_{0}\left|\mathrm{q}_{\mathrm{s}}\right|^{2}+\left|\mathrm{q}_{\mathrm{p}}\right|^{2}\right] \\
& \mathrm{C}^{+}(\sigma)=\left(\mathrm{k}_{\mathrm{s}}{ }^{3} / 2 \mathrm{k}_{0}\right)\left[\cos ^{2} \theta_{1}\left|\mathrm{q}_{\mathrm{s}}\right|^{2}+\left|\mathrm{q}_{\mathrm{p}}\right|^{2}\right]
\end{aligned}
$$

avec :

$$
\begin{aligned}
& \mathrm{q}_{\mathrm{s}}(\theta)=\mathrm{j}(2 \pi / \lambda) 2 \mathrm{n}_{0}\left(\mathrm{n}_{0}-\mathrm{n}_{1}\right)\left(\mathrm{n}_{0} \cos \theta_{0}+\mathrm{n}_{1} \cos \theta_{1}\right)^{-1} \\
& \mathrm{q}_{\mathrm{p}}(\theta)=\mathrm{j}(2 \pi / \lambda) 2 \mathrm{n}_{0}\left(\mathrm{n}_{0}-\mathrm{n}_{1}\right)\left(\mathrm{n}_{0} / \cos \theta_{0}+\mathrm{n}_{1} / \cos \theta_{1}\right)^{-1}
\end{aligned}
$$

et : $\quad \mathrm{n}_{0} \sin \theta_{0}=\mathrm{n}_{1} \sin \theta_{1}=\lambda \mathrm{v}=\mathrm{v} *$ qui est la fréquence normalisée.

On remarquera que ces formules sont aisées à programmer, de sorte qu'elles devraient venir systématiquement compléter les formules scalaires de la section 2, beaucoup moins riches d'information. En particulier on n'est plus limité par une valeur intégrée $\mathrm{S}$ de la diffusion (diffusion globale ou TIS), mais les formules ( $\underline{52-54})$ nous permettent de prédire la répartition angulaire des flux diffusés.

\subsection{Effets de polarisation}

L'onde diffusée présente des composantes TE (transverse électrique) et TM (transverse magnétique), souvent dénommées $\mathrm{S}$ et $\mathrm{P}$. Ces composantes sont définies en figure 7. Dans beaucoup de situations, on a besoin de connaître l'état de polarisation de la lumière diffusée. Celui-ci peut être obtenu par les formules ci-dessous :

$$
\begin{array}{ll}
I_{S S}=I \cos ^{2} \phi & I_{S P}=I \sin ^{2} \phi \\
I_{P P}=I \cos ^{2} \phi & I_{P S}=I \sin ^{2} \phi
\end{array}
$$

où $\mathrm{I}(\theta)$ désigne la diffusion angulaire calculée en lumière naturelle (formules (52-54)), de révolution pour une surface isotrope sous incidence normale. La dénomination $\mathrm{I}_{\mathrm{XY}}$ est utilisée pour désigner la quantité de lumière polarisée Y (TE ou TM) que l'on obtient quand l'éclairement est polarisé X (TE ou TM). Notons que ces formules ( $\underline{55})$ décrivent la variation polaire de l'intensité diffusée et montrent clairement qu'il n'y a pas de changement de polarisation dans le plan d'incidence pour les modes TE 
et TM ; il s'agit là encore d'une propriété caractéristique des surfaces ou objets faiblement perturbés, que l'on peut discriminer en conséquence. On peut compléter les relations ( $\underline{55})$ par :

$$
\begin{aligned}
& \mathrm{I}_{\mathrm{S}}=\mathrm{I}_{\mathrm{SS}}+\mathrm{I}_{\mathrm{SP}} \quad \mathrm{I}_{\mathrm{P}}=\mathrm{I}_{\mathrm{PP}}+\mathrm{I}_{\mathrm{PS}} \\
& \mathrm{I}_{\mathrm{LN}}=0,5\left(\mathrm{I}_{\mathrm{S}}+\mathrm{I}_{\mathrm{P}}\right)=0,5\left(\mathrm{I}_{\mathrm{SS}}+\mathrm{I}_{\mathrm{SP}}+\mathrm{I}_{\mathrm{PS}}+\mathrm{I}_{\mathrm{PP}}\right)
\end{aligned}
$$

où $\mathrm{LN}$ désigne le cas de la lumière naturelle.

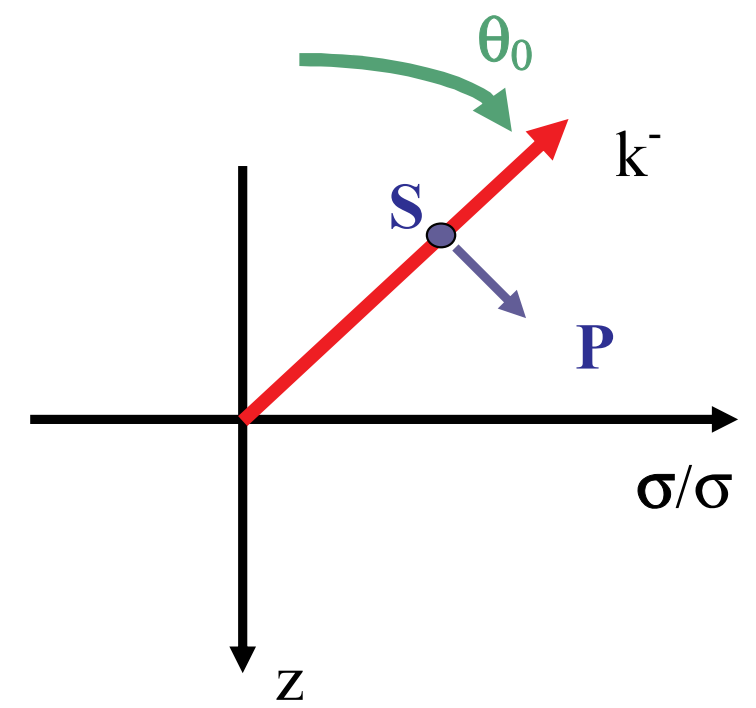

FIG. 7. Définition de la polarisation de l'onde diffusée dans le plan polaire.

\subsection{Spectre de rugosité}

Comme on l'a vu, en lumière naturelle et sous incidence normale, les coefficients $\mathrm{C}^{+-}$ne dépendent que de l'angle normal $\theta$. En conséquence c'est l'isotropie ou la non isotropie de la surface, décrite par la dépendance polaire du spectre de rugosité, qui impose les variations polaires de la diffusion. Il faut donc vérifier, de façon générale, que les techniques de polissage ou de nettoyage ne viennent pas orienter la rugosité des surfaces optiques. De façon analogue, la dépendance normale est imposée par le spectre de rugosité qui peut être décrit par différentes fonctions analytiques connues. L'expérience a montré que les fonctions d'autocorrélation des surfaces optiques isotropes pouvaient être approximées par la somme $\Gamma^{*}$ de gaussiennes $\Gamma_{\mathrm{g}}$ et d'exponentielles $\Gamma_{\mathrm{e}}$ :

$$
\Gamma^{*}=\Gamma_{\mathrm{e}}+\Gamma_{\mathrm{g}}=\delta_{\mathrm{e}}^{2} \exp \left[-\left|\tau / \mathrm{L}_{\mathrm{e}}\right|\right]+\delta_{\mathrm{g}}{ }^{2} \exp \left[-\left(\tau / \mathrm{L}_{\mathrm{g}}\right)^{2}\right]
$$

Après TF, on a : $\quad \gamma^{*}=\mathrm{TF}\left(\Gamma^{*}\right)=\gamma_{\mathrm{e}}+\gamma_{\mathrm{g}}$

$$
\text { avec : } \quad \begin{array}{ll}
\gamma_{\mathrm{e}}=(1 / 2 \pi)\left(\delta_{\mathrm{e}} \mathrm{L}_{\mathrm{e}}\right)^{2}\left[1+\left(\sigma \mathrm{L}_{\mathrm{e}}\right)^{2}\right]^{-3 / 2} \\
& \gamma_{\mathrm{g}}=(1 / 4 \pi)\left(\delta_{\mathrm{g}} \mathrm{L}_{\mathrm{g}}\right)^{2} \exp \left[-\left(\sigma \mathrm{L}_{\mathrm{g}} / 2\right)^{2}\right]
\end{array}
$$

Précisons que ces longueurs de corrélation sont proches de $: \mathrm{L}_{\mathrm{g}} \approx 0,1 \mu \mathrm{m}$ et $\mathrm{L}_{\mathrm{e}} \approx 2 \mu \mathrm{m}$ pour les surfaces polies. Pour des surfaces plus rugueuses, on peut se contenter de gaussiennes avec de faibles longueurs de corrélation qui conduisent à un flux angulaire quasi-constant dans tout le domaine angulaire. L'influence du spectre de rugosité sur la dépendance angulaire de l'indicatrice de diffusion est illustrée en figure 8. 


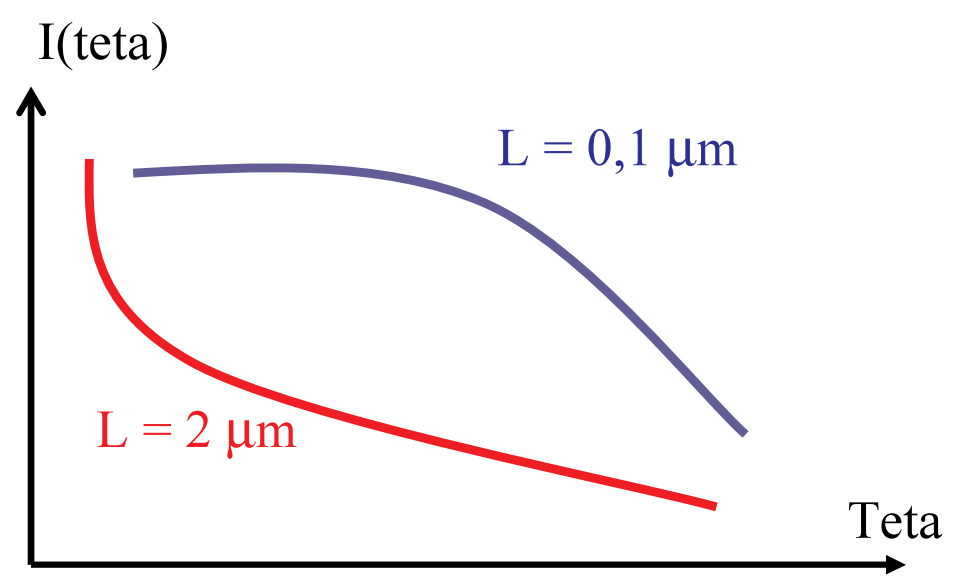

FIG. 8. Influence de la longueur de corrélation du spectre sur l'indicatrice de diffusion d'une surface.

On notera que les spectres $\gamma^{*}$ sont analytiques (et à dérivée lente) parce qu'ils représentent une moyenne du spectre intrinsèque $\gamma$ sur l'angle solide $\Delta \Omega$ de mesure du détecteur :

$$
\gamma^{*}(\sigma)=\int_{\Delta \Omega} \gamma(\sigma) \mathrm{d} \sigma
$$

Leur utilisation suppose donc que l'on étudie la diffusion avec une faible résolution angulaire, et que l'on ne cherche pas à se rapprocher de la résolution du speckle. Dès lors que l'on réduit ces effets de fonction d'appareil (divergence du faisceau ou taille de spot, angle solide...), il faut abandonner ces expressions et simuler numériquement les spectres, afin de retrouver les oscillations caractéristiques des flux mesurés à haute résolution angulaire. Précisons encore que les expressions ( $\underline{58})$ sont valables pour des surfaces isotropes, mais aussi pour les spectres moyens $\gamma_{m}$ (voir formule $(\underline{40-b})$ ) des surfaces anisotropes, quelle que soit la résolution utilisée. Cette remarque souligne l'importance et l'usage des expressions ( $\underline{58})$. Enfin, on peut traiter de la diffusion par un défaut isolé, ou d'une distribution déterministe de défauts, en calculant directement le spectre de ces défauts. On assiste alors à des oscillations dans le flux diffusé, analogues à celles données par les fonctions de Bessel...

\subsection{Conclusion}

Pour conclure, on veut insister sur la simplicité de codage numérique des expressions (53-54) qui donnent accès à la répartition angulaire des flux diffusés selon la loi :

$$
\mathrm{I}=\mathrm{C} \gamma
$$

et qui pourraient être utilisées de façon plus intensive. Rappelons que pour établir ces résultats, nous avons supposé la surface faiblement rugueuse et à faible pente :

$$
\delta / \lambda<1 \quad \text { et } \quad|\operatorname{grad} \mathrm{h}|<<1
$$

En fait chacun pourra vérifier que, dans le domaine des basses fréquences associées aux ondes planes et donc au calcul du champ lointain, une surface faiblement rugueuse ne peut pas avoir de fortes pentes, et inversement. Ainsi rugosité et pente sont liées dans la fenêtre fréquentielle optique, de sorte que le domaine de validité de ces théories du premier ordre se réduit à :

$$
\delta / \lambda<<1
$$


Enfin, ces théories se généralisent aux systèmes multicouches (피.99) de la façon suivante :

$$
\mathrm{I}=\Sigma \mathrm{C}_{\mathrm{ij}} \alpha_{\mathrm{ij}} \gamma_{\mathrm{j}}
$$

où I désigne le flux angulaire diffusé par le multicouche, $\mathrm{C}_{\mathrm{ij}}$ est un coefficient idéal lié à la formule du composant, $\gamma_{\mathrm{j}}$ le spectre de l'interface (j) dans le multicouche, et $\alpha_{\mathrm{ij}}$ le coefficient d'intercorrélation entre les profils des surfaces i et $\mathrm{j}$. Ce coefficient d'intercorrélation est lié à la cohérence mutuelle entre les multiples sources de diffusion dans l'empilement; lorsque sa valeur est élevée, on peut assister à des effets d'inhibition ou d'exaltation de la diffusion. On remarquera enfin que ce problème est strictement équivalent à celui des sources dans les microcavités planaires, à condition que sources et champs soient indépendants (hypothèse de champ faible). En effet, le problème de la diffusion lumineuse revient à calculer le champ rayonné par des courants électriques et magnétiques, surfaciques et volumiques, localisés dans des systèmes multicouches.

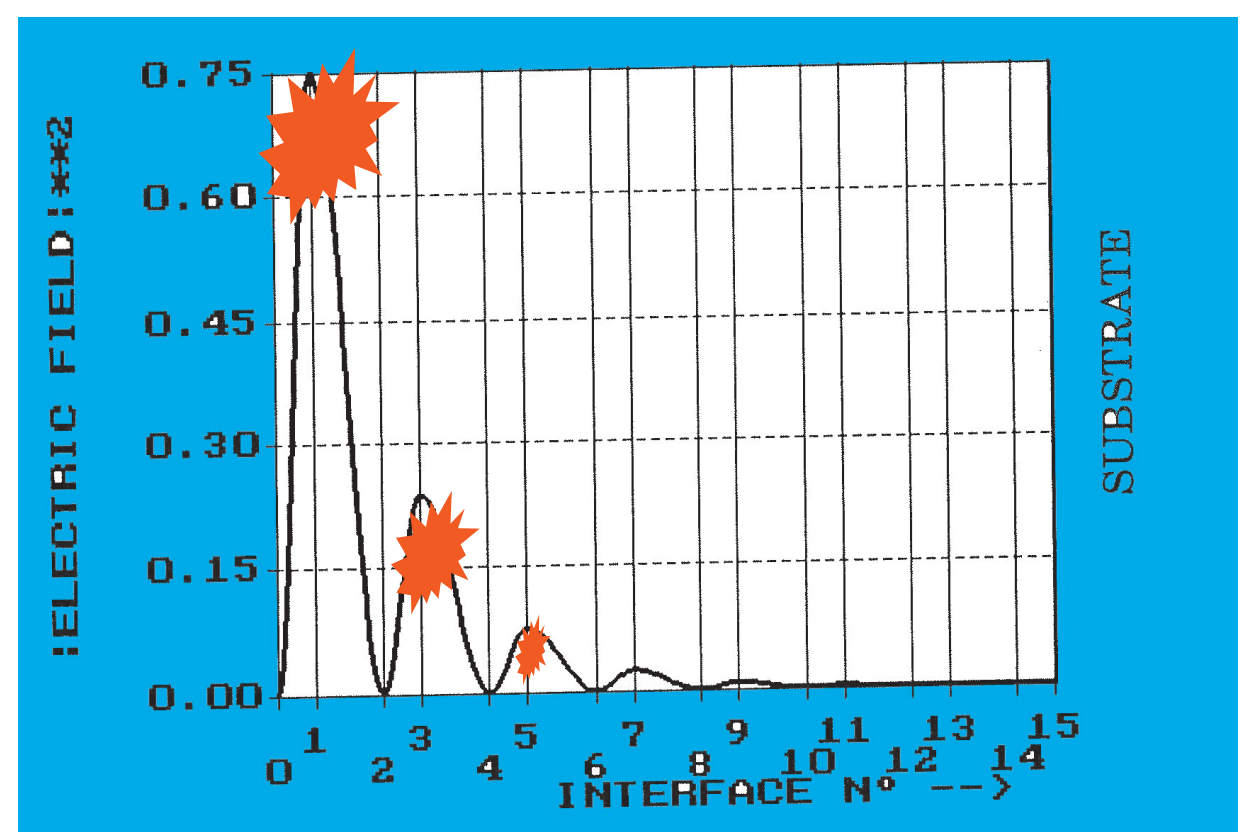

FIG. 9-a. Dioptres diffusants à l'intérieur d'un miroir multicouche quart d'onde.
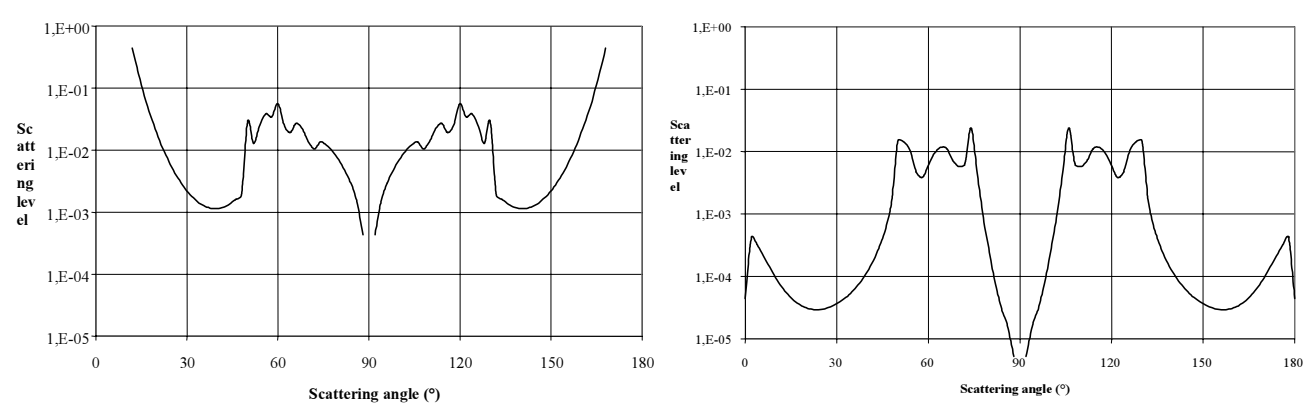

FIG. 9-b. Exemple de calcul de diffusion dans un filtre à bande étroite, pour des interfaces décorrélées (à gauche) et corrélées (à droite) (réalisation C. Deumié). 


\section{COMPARAISON DES MODÈLES SCALAIRE ET ÉLECTROMAGNÉTIQUE}

Nous avons montré comment utiliser la formule scalaire du TIS :

$$
\mathrm{S} / \mathrm{R}=(4 \pi \delta / \lambda)^{2}
$$

qui est une grandeur intégrée sur tout l'espace, ainsi que la formule de la diffusion angulaire :

$$
\mathrm{I}=\mathrm{C} \gamma
$$

qui est directionnelle. Il est intéressant de comparer ces expressions et voir dans quelle mesure elles peuvent conduire à des résultats équivalents.

Remarquons tout d'abord que, pour un dioptre unique, le coefficient $\mathrm{C}$ est quasiment constant sur tout le domaine angulaire $\left(0^{\circ}-90^{\circ}\right)$. On peut donc le remplacer par sa valeur à l'origine, ce qui nous donne :

$$
\begin{aligned}
& \mathrm{I}^{-}\left(\theta_{0}\right)=4 \mathrm{R}\left(2 \pi \mathrm{n}_{0} / \lambda\right)^{4} \gamma(\sigma) \\
& \mathrm{I}^{+}\left(\theta_{1}\right)=4 \mathrm{R} \mathrm{n}_{\mathrm{s}}{ }^{3} \mathrm{n}_{0}(2 \pi / \lambda)^{4} \gamma(\sigma)
\end{aligned}
$$

Ces dernières expressions sont encore plus faciles d'accès et peuvent rendre de précieux services. Si maintenant on les utilise pour calculer la diffusion globale S comme :

$$
S=\int I^{+-}(\theta, \phi) \sin \theta d \theta d \phi
$$

et que l'on néglige les variations des fonctions trigonométriques dans le domaine angulaire $\left(0^{\circ}-90^{\circ}\right)$, chacun pourra vérifier que l'on retrouve alors les formules scalaires du TIS. En conséquence les formules scalaires prennent tout leur sens lorsque les surfaces sont à faibles pentes et diffusent donc la majorité de la lumière au voisinage immédiat des faisceaux spéculaires.

\section{CAS DE LA DIFFUSION VOLUMIQUE}

Le cas de la diffusion volumique se traite de façon analogue, même si les paramètres statistiques, hypothèses et méthodes de résolution ne sont pas les mêmes. Les résultats obtenus demeurent simples, bien que le modèle théorique soit plus complexe.

\subsection{Paramètres de volume}

Le problème posé est illustré en figure 10. On considère deux milieux de permittivités $\varepsilon_{0}$ et $\varepsilon_{1}{ }_{1}$ séparés par l'interface plane $\mathrm{z}=0$. Le milieu incident ou superstrat $\left(\varepsilon_{0}, \mathrm{z}<0\right)$ est homogène avec une permittivité constante, tandis que le milieu substrat $\left(\varepsilon^{\prime}{ }_{1}, \mathrm{z}>0\right)$ supporte des hétérogénéités de volume, caractérisées par des fluctuations aléatoires d'indice de réfraction. La permittivité du milieu hétérogène est ainsi décrite par :

$$
\varepsilon_{1}^{\prime}(\mathbf{r}, \mathrm{z})=\varepsilon_{1}[1+\mathrm{p}(\mathbf{r}, \mathrm{z})]
$$

où $\mathrm{p}(\mathbf{r}, \mathrm{z})$ décrit les variations relatives de permittivité $(\varepsilon)$ ou d'indice de réfraction $(\mathrm{n})$ :

$$
\mathrm{p}(\mathbf{r}, \mathrm{z})=\left(\varepsilon^{\prime}{ }_{1}-\varepsilon_{1}\right) / \varepsilon_{1}=\Delta \varepsilon / \varepsilon=2 \Delta \mathrm{n} / \mathrm{n}
$$

Pour simplifier notre présentation, on supposera que les variations de permittivité sont uniquement transverses, ce qui permet de traiter une grande majorité de problèmes. On écrira donc :

$$
\mathrm{p}(\mathbf{r}, \mathrm{z})=\mathrm{p}(\mathbf{r})
$$




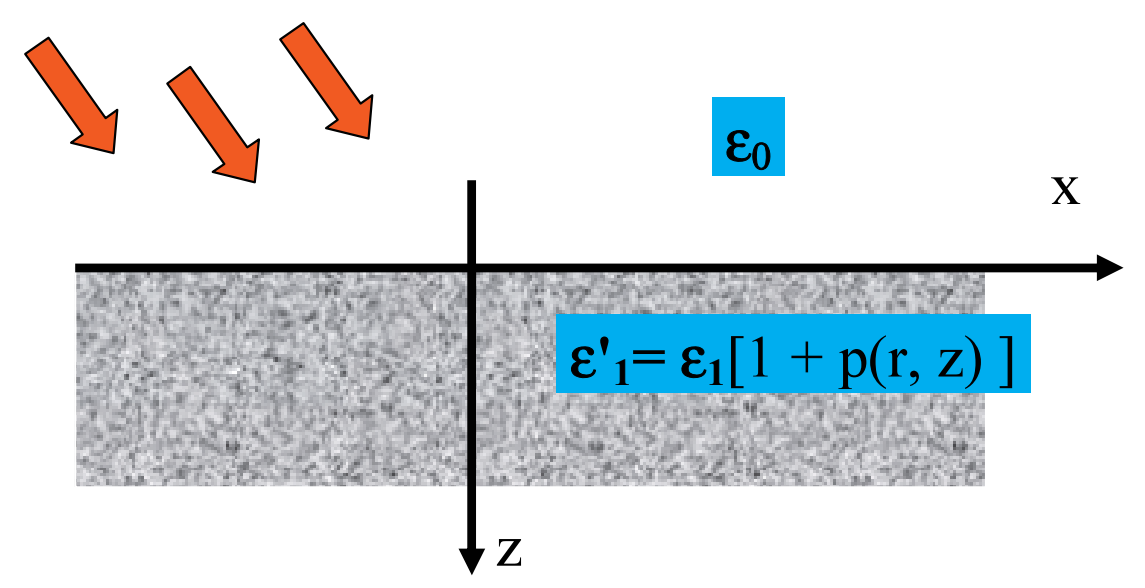

FIG. 10. Volume hétérogène.

Comme pour la rugosité, on utilisera les grandeurs classiques suivantes pour décrire et quantifier l'hétérogénéité d'indice à partir de la fonction $\mathrm{p}$ :

$$
\begin{aligned}
& \text { - moyenne : } \quad \mathrm{p} / 2=(1 / \mathrm{S}) \int_{\mathbf{r}}(\Delta \mathrm{n} / \mathrm{n})(\mathbf{r}) \mathrm{d} \mathbf{r}=(1 / 2)(1 / \mathrm{S}) \int_{\mathbf{r}} \mathrm{p}(\mathbf{r}) \mathrm{d} \mathbf{r} \\
& \text { - écart-type : } \quad \delta_{\mathrm{v}}{ }^{2}=(1 / \mathrm{S}) \int_{\mathbf{r}}(\Delta \mathrm{n} / \mathrm{n})^{2}(\mathbf{r}) \mathrm{d} \mathbf{r}=(1 / 4)(1 / \mathrm{S}) \int_{\mathbf{r}} \mathrm{p}^{2}(\mathbf{r}) \mathrm{d} \mathbf{r} \\
& \text { - fonction d'autocorrélation : } \Gamma(\tau)=(1 / \mathrm{S}) \int_{\mathbf{r}} \mathrm{p}(\mathbf{r}) \mathrm{p}(\mathbf{r}-\tau) \mathrm{d} \mathbf{r} \text { avec } \Gamma(\mathbf{0}) / 4=\delta_{\mathrm{v}}{ }^{2} \\
& \text { - spectre de permittivité : } \quad \gamma(\boldsymbol{\sigma})=\left(4 \pi^{2} / \mathrm{S}\right)|p(\boldsymbol{\sigma})|^{2} \\
& \text { avec : (1/4) } \int_{\boldsymbol{\sigma}} \gamma(\boldsymbol{\sigma}) \mathrm{d} \boldsymbol{\sigma}=\delta_{\mathrm{v}}{ }^{2}
\end{aligned}
$$

On remarquera, par opposition à la diffusion de surface, que la fonction d'autocorrélation de la permittivité est sans dimension, de sorte que le spectre de permittivité a pour dimension le $\left[\mathrm{m}^{2}\right]$. De façon générale, les défauts volumiques ont une faible longueur de corrélation (de l'ordre du dixième de micron) et sont souvent approximés à l'aide d'une gaussienne :

$$
\begin{aligned}
& \Gamma^{*}=\delta_{\mathrm{g}}{ }^{2} \exp \left[-\left(\tau / \mathrm{L}_{\mathrm{g}}\right)^{2}\right] \\
\Rightarrow \quad & \gamma^{*}=\mathrm{TF}\left(\Gamma^{*}\right)=\gamma_{\mathrm{g}}=(1 / 4 \pi)\left(\delta_{\mathrm{g}} \mathrm{L}_{\mathrm{g}}\right)^{2} \exp \left[-\left(\sigma \mathrm{L}_{\mathrm{g}} / 2\right)^{2}\right]
\end{aligned}
$$

\subsection{Modèle électromagnétique}

Comme pour la diffusion de surface, le champ volumique diffusé $\mathbf{E}^{\mathbf{d}}$ est défini comme la différence entre le champ total $\mathbf{E}$ ré-émis par le volume hétérogène et le champ idéal $\mathbf{E}^{\mathbf{0}}$ qui existerait pour un volume parfaitement homogène :

$$
\mathbf{E}^{\mathbf{d}}=\mathbf{E}-\mathbf{E}^{\mathbf{0}}
$$

Ces champs $\mathbf{E}$ et $\mathbf{E}^{\mathbf{0}}$ vérifiant les équations de Maxwell au sens des fonctions dans les 2 milieux avec les couples de permittivités $\left(\varepsilon_{0}, \varepsilon^{\prime}\right)$ et $\left(\varepsilon_{0}, \varepsilon_{1}\right)$ respectivement, on vérifie facilement que l'on a :

$$
\begin{aligned}
& \operatorname{rot} \mathbf{E}_{i}^{d}=j \omega \mu \mathbf{H}_{i}{ }^{d} \\
& \operatorname{rot}{\mathbf{H}_{i}}^{\mathbf{d}}=-j \omega \varepsilon_{i} \mathbf{E}_{i}^{d}+\mathbf{J}(\mathbf{r}, z)
\end{aligned}
$$


où $\mathbf{J}(\mathbf{r}, \mathrm{z})=-\mathrm{j} \omega \varepsilon_{1} \mathrm{p}(\mathbf{r}) \mathbf{E}(\mathbf{r}, \mathrm{z})$ joue le rôle d'un courant électrique volumique. À ce stade, on se place à nouveau dans l'hypothèse de l'approximation de Born, pour ne considérer que les termes du premier ordre. Cela revient à considérer que le courant $\mathbf{J}$ est uniquement excité par le champ idéal $\mathbf{E}^{\mathbf{0}}$ :

$$
\mathbf{J}(\mathbf{E})=\mathbf{J}\left(\mathbf{E}^{\circ}\right)
$$

Cette hypothèse n'est évidemment validée que pour des milieux faiblement hétérogènes, présentant une faible diffusion volumique :

$$
|\mathrm{p}(\mathbf{r})|=|\Delta \varepsilon / \varepsilon|=2|\Delta \mathrm{n} / \mathrm{n}|<<1
$$

Les sources étant ainsi connues, il ne reste plus qu'à résoudre le système $(\underline{78})$ avec les outils adéquats. En particulier on utilisera les fonctions ou matrices de Green dans le plan complexe, conjointement à la notion d'admittance complexe. On obtient alors une expression similaire à celle de la diffusion de surface, à savoir :

$$
\mathrm{I}(\theta, \phi)=\mathrm{C}(\theta, \phi) \gamma(\theta, \phi)
$$

où I est l'intensité diffusée dans la direction $(\theta, \phi)$ et $\mathrm{C}$ désigne un facteur optique indépendant de la microstructure du volume hétérogène, mais qui varie avec les indices moyens des milieux, les conditions d'incidence et de polarisation. Le terme $\gamma$, quant à lui, contient toute l'information accessible sur les hétérogénéités de volume, et représente le spectre de permittivité du volume $\varepsilon_{1}^{\prime}$ :

$$
\gamma(\boldsymbol{\sigma})=\left(4 \pi^{2} / \mathrm{S}\right)|p(\boldsymbol{\sigma})|^{2}
$$

Comme pour la diffusion de surface, la formule ( $\underline{81})$ se généralise rapidement au cas des systèmes multicouches, filtres ou guides ou microcavités. On insistera, dans le cas d'un volume unique, sur le fait que la formule ( $\underline{81})$, parce qu'elle permet d'extraire directement de la mesure le spectre de permittivité, fournit une méthode unique pour caractériser les hétérogénéités d'indice avec des paramètres optiques.

\subsection{Comparaison des diffusions de surface et de volume}

De façon générale, un problème récurrent se pose quant à la séparation des diffusions de surface et de volume. En d'autres termes, lorsqu'on effectue une mesure de flux diffusé en champ lointain, est-il possible d'identifier l'origine de cette diffusion (surface ou volume) ? Plusieurs techniques ont été développées dans ce but :

- le dépôt de couches métalliques opaques permet d'isoler une interface par rapport au volume ; toutefois il faut s'assurer de la parfaite réplication de l'interface rugueuse par la couche déposée ;

- le dépôt d'une couche mince diélectrique permet également, grâce à un effet «antidiffusant », de réduire voire annuler la diffusion de surface pour mieux révéler la diffusion de volume ; 
- l'anisotropie de la diffusion est souvent une signature de l'état de surface, contrairement aux volumes qui rayonnent en général un flux isotrope ;

- les variations spectrales (avec la longueur d'onde) de la diffusion sont différentes selon l'origine de la diffusion. En particulier la diffusion de volume est en phase avec la transmission, alors que la diffusion de surface est en phase avec la réflexion pour des interfaces corrélées ;

- enfin, les taux de dépolarisation de la lumière diffuse sont caractéristiques de la présence de rugosités de surface ou d'hétérogénéités de volume, et permettent donc de discriminer ces effets. C'est pourquoi l'ellipsométrie sur champ diffus dans chaque direction de l'espace apparaît comme une technique prometteuse pour aller plus loin dans ces investigations. En particulier le déphasage polarimétrique de l'onde diffusée ne dépend souvent que de l'origine de la diffusion.

Pour donner un ordre de grandeur des diffusions de surface et de volume, une rugosité de $0,5 \mathrm{~nm}$ et une hétérogénéité d'indice de $10^{-3}$ créent des diffusions angulaires équivalentes pour un substrat de silice.

\section{MESURE DE LA DIFFUSION}

On trouvera une description détaillée de la mesure de diffusion dans plusieurs références. Le cas des sphères intégrantes ( $\underline{\text { Fig. 11-a }}$ ) est très connu et pratique, et donne accès à la mesure immédiate du TIS intégré dans le domaine angulaire complémentaire des ouvertures de la sphère. Dans le cas de la diffusion angulaire (Fig. 11-b), l'instrumentation est a priori simple à mettre en oeuvre, lorsque les surfaces sont fortement diffusantes (flux angulaires supérieurs à $10^{-4}$ ). Par contre, dès que l'on s'intéresse à des composants de qualité optimale, présentant des pertes inférieures à $10^{-6}$, la mesure est plus délicate et il faut résoudre les problèmes de lumière parasite. Celle-ci provient tout d'abord de la divergence et diffraction qui accompagne le faisceau incident en raison des nombreuses optiques et diaphragmes traversés avant éclairement de la surface à étudier. La lumière parasite est également créée par les particules en suspension dans l'air, et qui viennent diffracter, selon leur taille, au voisinage immédiat ou secondaire des faisceaux spéculaires. De façon générale, la mesure des faibles flux diffusés au voisinage immédiat des faisceaux spéculaires demeure un problème difficile. A titre d'illustration, un exemple d'appareillage et de mesure est présenté en figure $\mathbf{1 2}$.

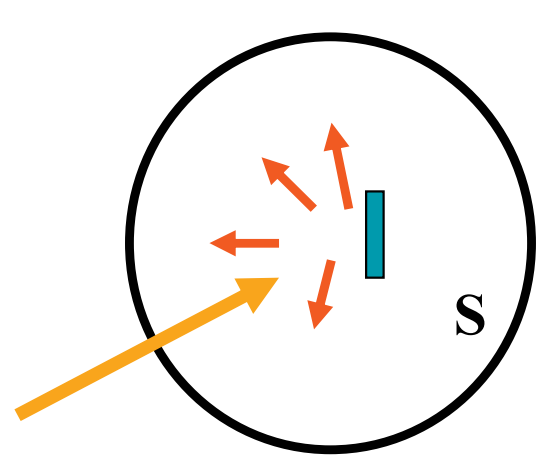

FIG. 11-a. Sphère intégrante.

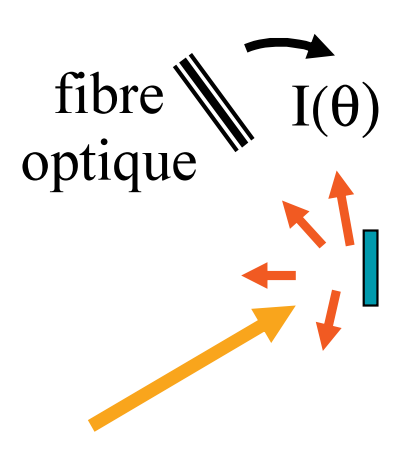

FIG. 11-b. Diffusomètre angulaire. 


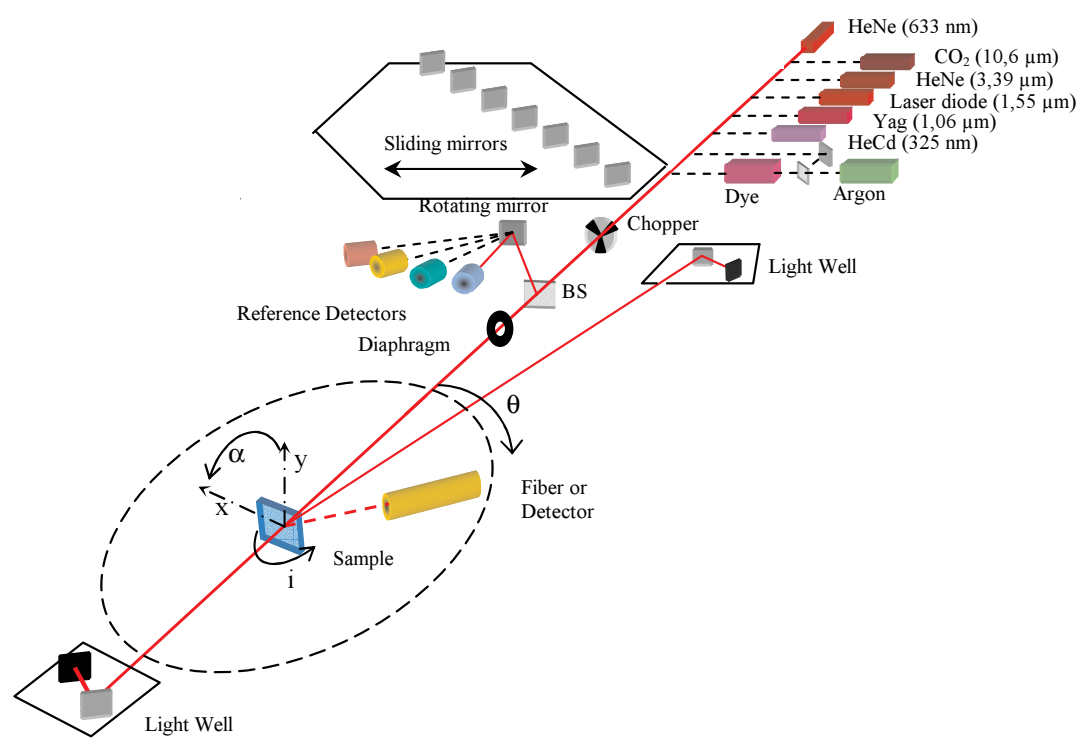

FIG. 12-a. Exemple de diffusomètre angulaire développé à l'Institut Fresnel (10 axes motorisés, $325 \mathrm{~nm}-10,6 \mu \mathrm{m})$.

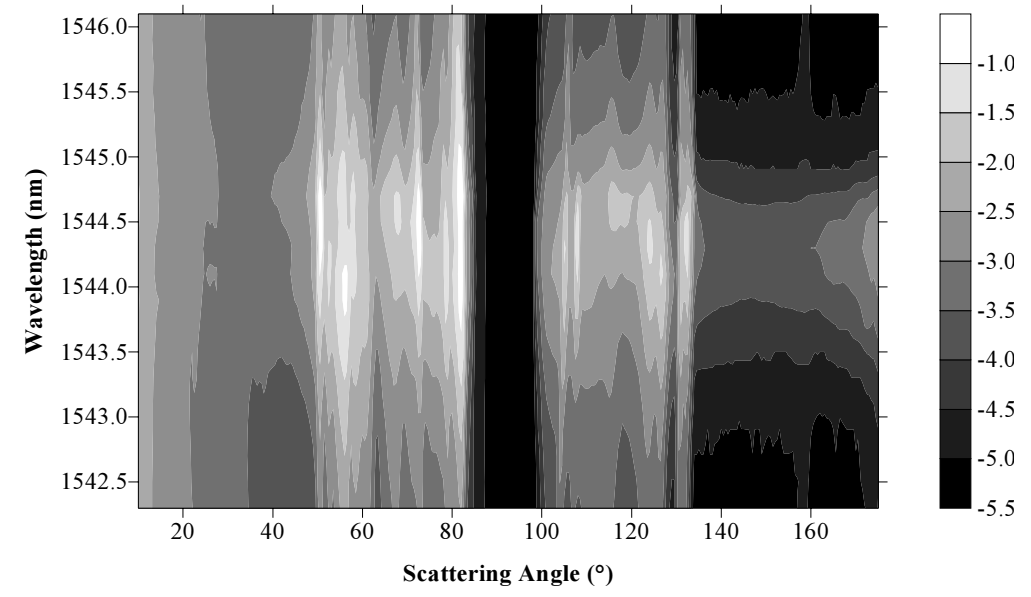

FIG. 12-b. Mesure angulaire et spectrale de diffusion dans un filtre WDM bande étroite (réalisation C. Deumié/M. Lequime, Institut Fresnel). 


\section{FENÊTRE FRÉQUENTIELLE OPTIQUE}

Nous avons vu que les techniques de diffusion angulaire permettaient de caractériser la microstructure (rugosité, hétérogénéités...) des surfaces et volumes de composants. Toutefois cette caractérisation s'effectue dans une certaine fenêtre de fréquences spatiales ou bande passante donnée par :

$$
\left.\mathrm{B}\left(\theta_{\mathrm{m}}, \lambda\right)=\right] \sin \theta_{\mathrm{m}} / \lambda, 1 / \lambda[
$$

où $\lambda$ est la longueur d'onde d'étude et $\theta_{\mathrm{m}}$ l'angle minimum de mesure de la diffusion. Ainsi la borne maximale de la bande passante est donnée par l'inverse de la longueur d'onde, au delà de laquelle les ondes sont évanescentes, et la borne minimale est liée à l'angle minimum de mesure où diffusion et réflexion se superposent. En conséquence les paramètres de rugosité ou d'hétérogénéité, comme toute grandeur physique mesurée, sont des grandeurs relatives, puisqu'elles sont obtenues par intégration d'un spectre de rugosité ou de permittivité dans une bande passante donnée. Ceci explique, par exemple, pourquoi la rugosité dépend de la longueur d'onde; on n'utilisera donc pas des valeurs mesurées dans le visible pour prédire des pertes dans l'infra-rouge. Comme illustré en figure 13, on peut considérer la planéité comme une rugosité que l'on obtiendrait dans le lointain IR, alors qu'une rugosité mesurée avec une source X-UV se rapproche des paramètres microscopiques obtenues par les microscopes à champ proche ou sonde locale.

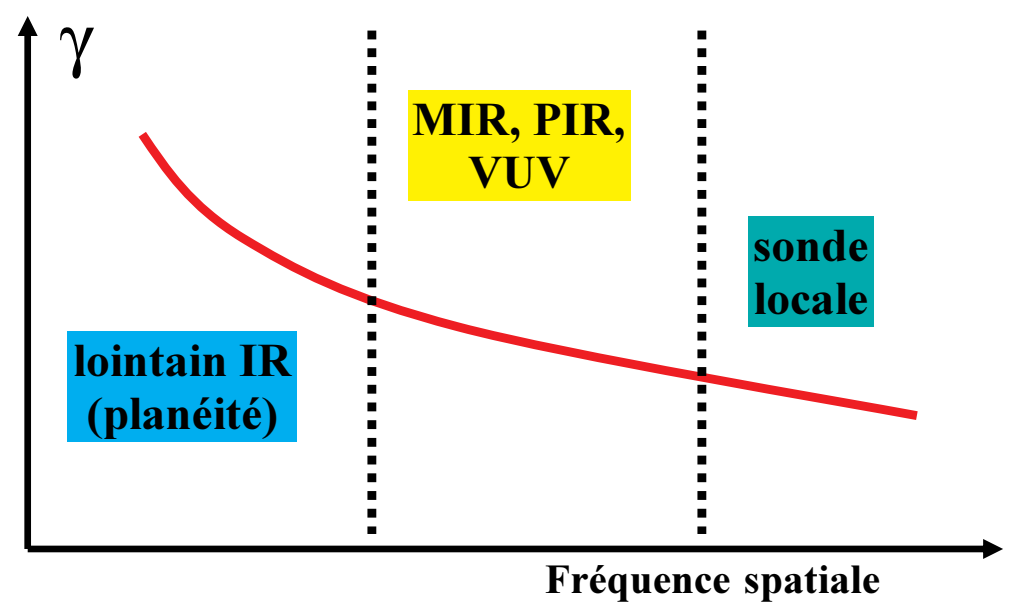

FIG. 13. Du macroscopique au microscopique.

Si l'on veut maintenant comparer les valeurs de rugosité (macroscopiques) obtenues par diffusion lumineuse, et celles (microscopiques) obtenues par microscopie à sonde locale (microscopie à force atomique, à effet tunnel optique...), il est nécessaire de spécifier la bande fréquentielle des techniques d'échantillonnage de type AFM ou PSTM :

$$
\mathrm{B}(\mathrm{L}, \Delta \mathrm{x})=] 1 / 2 \mathrm{~L}, 1 / 2 \Delta \mathrm{x}[
$$

où $\mathrm{L}$ est la dimension de la surface imagée et $\Delta \mathrm{x}=\mathrm{L} / \mathrm{N}$ le pas d'échantillonnage, avec $\mathrm{N}$ le nombre de points. On voit donc que les techniques sont à priori comparables à condition d'imposer la condition : $\mathrm{L} \approx \Delta \mathrm{x}$, c'est-à-dire un pas d'échantillonnage pour l'AFM égal à la longueur d'onde pour l'optique. Toutefois ces techniques ne sont pas faites pour fonctionner dans la même fenêtre fréquentielle, l'optique étant en général réservée aux grandeurs macroscopiques et l'AFM aux grandeurs microscopiques. En particulier les problèmes de stationnarité sont cruciaux lors des problèmes d'intercomparaison. 


\section{CONCLUSION}

Les techniques de diffusion lumineuse sont bien adaptées pour sonder en champ lointain (de façon non destructrice) les surfaces et volumes des composants. Lorsque les objets sont faiblement hétérogènes, la simplicité des outils théoriques fournit une méthode directe et simple d'accès pour quantifier de façon unique les rugosités et en particulier les hétérogénéités d'indice, avec une précision relative de l'ordre du pour-cent. De façon plus générale, la dépendance normale et polaire de la diffusion, ses variations spectrales, ou le déphasage polarimétrique, le contraste du speckle..., permettent de discriminer nombre d'hétérogénéités particulières enfouies dans les composants interférentiels; en ce sens, les méthodes de diffusion apparaissent aujourd'hui encore, sousexploitées. Au delà de l'optique de précision, les champs d'application couvrent la vision, la cosmétique, l'éclairage..., avec des méthodes qui se généralisent à la télédétection, l'imagerie polarimétrique et en milieu diffus...

\section{RÉFÉRENCES}

L'expertise acquise pour ce cours résulte essentiellement des activités de recherche développées à l'Institut Fresnel depuis plus de 25 ans, en particulier grâce à Paul Bousquet et Pierre Roche qui ont été initiateurs dans le domaine. L'instrument de mesure et la modélisation ont progressé grâce à la contribution de nombreuses thèses (D. Torricini, L. Bruel, F. Cleva, S. Maure et C. Deumié...).

1. J.M. Eatsman, Surface scattering in optical interference coatings, Ph. D. thesis, University of Rochester, New-York (1974).

2. P. Croce et L. Prod'homme, Etude par diffusion lumineuse de la nature des surfaces de verre poli, Nouv. Rev. Optique 7 (2) (1976) 121-132.

3. C.K. Carniglia, Scalar theory for multilayer optical coatings, Opt. Eng. 18 (1979) 104-115.

4. J.M. Elson et J.M. Bennett, Relation between the angular dependence of scattering and the statistical properties of optical surfaces, J. Opt. Soc. Am. 69 (1979) 31-47.

5. J.M. Elson, J.P. Rahn et J.M. Bennet, Light scattering from multilayer optics: comparison of theory and experiment, Appl. Opt. 19 (1980) 669-679.

6. P. Bousquet, F. Flory et P. Roche, Scattering from multilayer thin films: theory and experiment, J. Opt. Soc. Am. 71 (1981) 1115-1123.

7. J.M. Elson, J.P. Rahn et J.M. Bennett, Relationship of the total integrated scattering from multilayer-coated optics to angle of incidence, polarization, correlation length, and roughness cross-correlation properties, Appl. Opt. 22 (1983) 3207-3219.

8. J.M. Elson, Theory of light scattreing from a rough surface with an inhomogeneous permittivity, Phys. Rev. B 30 (1984) 5460-5480.

9. P. Roche et E. Pelletier, Characterization of optical surfaces by measurement of scattering distribution, Appl. Opt. 23 (1984) 3561-3566.

10. C. Amra, G. Albrand et P. Roche, Theory and application of antiscattering single layers; antiscattering antireflection coatings, Appl. Opt. 25 (1986) 2695-2702.

11. J. M. Bennet et L. Mattsson, Introduction to surface roughness and scattering, Optical Society of America, Washington, D. C. (1989).

12. C. Amra, J.H. Apfel et E. Pelletier, The role of interface correlation in light scattering by a multilayer, Appl. Opt. 31 (1992) 3134-3151.

13. S. Kassam, A. Duparré, K. Hehl, P. Bussemer et J. Neubert, Light scattering from the volume of optical thin films: theory and experiment, Appl. Opt. 31 (1992) 1304-1313. 
14. A. Duparré et S. Kassam, Relation between light scattering and microstructure of optical thin films, Appl. Opt. 32 (1992) 5475-5480.

15. C. Amra, First order vector theory of bulk scattering in optical multilayers, J. Opt. Soc. Am. A 10 (1993) 365-374.

16. C. Amra, C. Grèzes-Besset et L. Bruel, Comparison of surface and bulk scattering in optical coatings, Appl. Opt. 32 (1993) 5492-5503.

17. C. Amra, From light scattering to the microstructure of thin film multilayers, Appl. Opt. 32 (1993) 5481-5491.

18. C. Amra, D. Torricini et P. Roche, Multiwavelength $(0.45-10.6 \mu \mathrm{m})$ angle-resolved scatterometer or how to extend the optical window, Appl. Opt. 32 (1993) 5462-5474.

19. C. Amra, Light scattering from multilayer optics - Part A: Investigation tools, J. Opt. Soc. Am. A 11 (1994) 197-210.

20. C. Amra, Light scattering from multilayer optics - Part B: Application to experiment, J. Opt. Soc. Am. A 11 (1994) 211-226.

21. C. Deumié, R. Richier, P. Dumas et C. Amra, Multiscale roughness in optical multilayers: atomic force microscopy and light scattering,. Appl. Opt. 35 (1996) 5583-5594.

22. S. Maure, G. Albrand, et C. Amra, Low-level scattering and localized defects, Appl. Opt. 35 (1996) 5573-5582.

23. C. Deumié, H. Giovannini et C. Amra, Ellipsometry of light scattering from thin film multilayer coatings,. Appl. Opt. 35 (1996) 5600-5608.

24. C. Amra et S. Maure, Electromagnetic power provided by sources within multilayer optics: free space and modal patterns, J. Opt. Soc. Am. A 14 (1997) 3102-3113.

25. C. Amra et S. Maure, Mutual coherence and conical pattern of sources optimally excited within multilayer optics, J. Opt. Soc. Am. A 14 (1997) 3114-3124.

26. H. Giovannini et C. Amra, Scattering reduction effect with overcoated rough surfaces: theory and experiment, Appl. Opt. 36 (1997) 5574-5579.

27. H. Giovannini et C. Amra, Dielectric thin films for maximized absorption with standard black quality surfaces, Appl. Opt. 37 (1998).

28. M. Lequime, C. Deumié et C. Amra, Light scattering from WDM filters, Advances in Optical Interference Coatings, SPIE 3738 (1999) 268-277.

29. P. Kadkhoda, C. Amra, J.M. Bennett, C. Deumié, A. Duparré et al., International round-robin experiment to test the ISO Total scattering draft standard, Appl. Opt. 39 (2000) 3321-3332.

30. C. Deumié, H. Giovannini et C. Amra, Angle-Resolved ellipsometry of light scattering: discremination of surface and bulk effects in substrates and optical coatings, Appl. Opt. 41 (2002) 3362-3369.

31. C. Deumié, P. Voarino et C. Amra, Overcoated microspheres for specific optical powders, Appl. Opt. 41 (2002) 3299-3305. 